\title{
THE REES VALUATIONS OF COMPLETE IDEALS IN A REGULAR LOCAL RING
}

\author{
WILLIAM HEINZER AND MEE-KYOUNG KIM
}

\begin{abstract}
Let $I$ be a complete $\mathbf{m}$-primary ideal of a regular local ring $(R, \mathbf{m})$ of dimension $d \geq 2$. In the case of dimension two, the beautiful theory developed by Zariski implies that $I$ factors uniquely as a product of powers of simple complete ideals and each of the simple complete factors of $I$ has a unique Rees valuation. In the higher dimensional case, a simple complete ideal of $R$ often has more than one Rees valuation, and a complete $\mathbf{m}$-primary ideal $I$ may have finitely many or infinitely many base points. For the ideals having finitely many base points Lipman proves a unique factorization involving special $*$-simple complete ideals and possibly negative exponents of the factors. Let $T$ be an infinitely near point to $R$ with $\operatorname{dim} R=\operatorname{dim} T$ and $R / \mathbf{m}=T / \mathbf{m}_{T}$. We prove that the special $*$-simple complete ideal $P_{R T}$ has a unique Rees valuation if and only if either $\operatorname{dim} R=2$ or there is no change of direction in the unique finite sequence of local quadratic transformations from $R$ to $T$. We also examine conditions for a complete ideal to be projectively full.
\end{abstract}

\section{INTRODUCTION}

Motivation for our work in this paper comes from an interesting article of Joseph Lipman $[\mathrm{L}$. Lipman considers the structure of a certain class of complete ideals, the finitely supported complete ideals, in a regular local ring (RLR) of dimension $d \geq 2$. He proves a factorization theorem for the finitely supported complete ideals that extends the factorization theory of complete ideals in a two-dimensional RLR as developed by Zariski [ZS2, Appendix 5]. Other work on this topic has been done by John Gately in [G1] and [G2, and by Campillo, Gonzalez-Sprinberg and Lejeune-Jalabert in CGL.

All rings we consider are assumed to be commutative with an identity element. We use the concept of complete ideals as defined and discussed in Swanson-Huneke [SH, Chapters 5,6,14]. We also use a number of concepts considered in Lipman's paper $[\mathrm{L}$. The product of two complete ideals in a two-dimensional regular local

Date: October 17, 2018.

1991 Mathematics Subject Classification. Primary: 13A30, 13C05; Secondary: 13E05, 13 H15.

Key words and phrases. Rees valuation, finitely supported ideal, special *-simple complete ideal, base points, point basis, transform of an ideal, local quadratic transform, projective equivalence, projectively full. 
ring is again complete. This no longer holds in higher dimension, $\mathrm{C}]$ or $[\mathrm{Hu}]$. To consider the higher dimensional case, one defines for ideals $I$ and $J$ the $*$-product, $I * J$ to be the completion of $I J$. A complete ideal $I$ in a commutative $\operatorname{ring} R$ is said to be $*$-simple if $I \neq R$ and if $I=J * L$ with ideals $J$ and $L$ in $R$ implies that either $J=R$ or $L=R$.

Another concept used by Zariski in [ZS2] is that of the transform of an ideal; the complete transform of an ideal is used in $[\mathrm{L}$ and [G2].

Definition 1.1. Let $R \subseteq T$ be unique factorization domains (UFDs) with $R$ and $T$ having the same field of fractions, and let $I$ be an ideal of $R$ not contained in any proper principal ideal.

(1) The transform of $I$ in $T$ is the ideal $I^{T}=a^{-1} I T$, where $a T$ is the smallest principal ideal in $T$ that contains $I T$.

(2) The complete transform of $I$ in $T$ is the completion $\overline{I^{T}}$ of $I^{T}$.

A proper ideal $I$ in a commutative ring $R$ is simple if $I \neq L \cdot H$, for any proper ideals $L$ and $H$. An element $\alpha \in R$ is said to be integral over $I$ if $\alpha$ satisfies an equation of the form

$$
\alpha^{n}+r_{1} \alpha^{n-1}+\cdots+r_{n}=0, \quad \text { where } \quad r_{i} \in I^{i}
$$

The set of all elements in $R$ that are integral over an ideal $I$ forms an ideal, denoted by $\bar{I}$ and called the integral closure of $I$. An ideal $I$ is said to be complete (or, integrally closed) if $I=\bar{I}$.

For an ideal $I$ of a local ring $(R, \mathbf{m})$, the order of $I$, denoted $\operatorname{ord}_{R} I$, is $r$ if $I \subseteq \mathbf{m}^{r}$ but $I \nsubseteq \mathbf{m}^{r+1}$. If $(R, \mathbf{m})$ is a regular local ring, the function that associates to an element $a \in R$, the order of the principal ideal $a R$, defines a discrete rank-one valuation, denoted $\operatorname{ord}_{R}$ on the field of fractions of $R$. The associated valuation ring (DVR) is called the order valuation ring of $R$.

Let $I$ be a nonzero ideal of a Noetherian integral domain $R$. The set of Rees valuation rings of $I$ is denoted Rees $I$, or by $\operatorname{Rees}_{R} I$ to also indicate the ring in which $I$ is an ideal. It is by definition the set of DVRs

$$
\left.\left\{\overline{\left(R\left[\frac{I}{a}\right]\right.}\right)_{Q} \mid \quad 0 \neq a \in I \quad \text { and } \quad Q \in \operatorname{Spec}\left(\overline{R\left[\frac{I}{a}\right]}\right) \quad \text { is of height one with } I \subset Q\right\} \text {, }
$$
where $\overline{-}$ denotes integral closure in the field of fractions. The corresponding discrete valuations with value group $\mathbb{Z}$ are called the Rees valuations of $I$. In general, if $J \subseteq I$ is a reduction, then we have Rees $J=\operatorname{Rees} I$. 
An ideal $I$ is said to be normal if all the powers of $I$ are complete. Let $I$ be a normal $\mathbf{m}$-primary ideal of a normal Noetherian local domain $(R, \mathbf{m})$. The minimal prime ideals of $\mathbf{m} R[I t]$ in the Rees algebra $R[I t]$ are in one-to-one correspondence with the Rees valuation rings of $I$. The correspondence associates to each Rees valuation ring $V$ of $I$ a unique prime $P \in \operatorname{Min}(\mathbf{m} R[I t])$ such that $V=R[I t]_{P} \cap \mathcal{Q}(R)$. Properties of the quotient ring $R[I t] / P$ relate to properties of certain birational extensions of $R$.

If $(R, \mathbf{m})$ is a two-dimensional regular local ring, then the Zariski theory implies that a simple complete $\mathbf{m}$-primary ideal has a unique Rees valuation ring. However, if the dimension of $R$ is greater than two, then a simple complete $\mathbf{m}$-primary ideal may have more than one Rees valuation ring; indeed, this is often the case even for a special $*$-simple complete ideal as in Definition 2.8. An ideal $I$ of a Noetherian integral domain $R$ is said to one-fibered if $I$ has a unique Rees valuation.

In the case where $(R, \mathbf{m})$ is a two-dimensional regular local ring, Zariski's unique factorization theorem implies that a complete $\mathbf{m}$-primary ideal $I$ can be factored uniquely as a finite product of powers of simple complete ideals. The distinct simple factors of $I$ are in one-to-one correspondence with the Rees valuation rings of $I$.

If $I$ is a simple complete ideal of a two-dimensional RLR and $R / \mathbf{m}$ is algebraically closed, Huneke and Sally [HS, Theorem 3.8] prove that $R[I t] / P$ is regular. This result is extended in $[\mathrm{K}$, Theorem 3.1] by proving that if $R / \mathbf{m}$ is algebraically closed, then $I$ is a product of distinct simple complete ideals if and only if $R[I t] / P$ is regular for each minimal prime $P$ of $\mathbf{m} R[I t]$.

Let $(R, \mathbf{m})$ be a regular local ring of dimension $d \geq 2$. In Section 2 we discuss the structure of regular local rings $T$ birational over $R$, and the order valuation ring of $T$. In Section 3 we review Lipman's unique factorization theorem and raise several questions about the base points of finitely supported complete ideals. Let $I$ be an m-primary ideal of $R$. In Section 4 we compare the Rees valuations of $I$ with the Rees valuations of the transform $I_{1}$ of $I$ in $S_{1}=R\left[\frac{\mathbf{m}}{x}\right]$, where $x \in \mathbf{m} \backslash \mathbf{m}^{2}$. We prove in Proposition 4.3 that Rees $I \subseteq \operatorname{Rees}_{S_{1}} I_{1} \cup$ Rees $\mathbf{m}$. If $I$ is finitely supported, we prove in Proposition 4.6 that $\operatorname{Rees}_{S_{1}} I_{1} \subseteq$ Rees $I$, and demonstrate in Example 4.9 that this may fail if $I$ is not finitely supported.

We observe in Remark 5.2 that every special $*$-simple complete ideal is projectively full. In Proposition 5.6 we prove that a complete $\mathbf{m}$-primary ideal of $R$ is projectively full if the transform $I_{1}$ of $I$ in $S_{1}$ is projectively full. In Section 6 we examine 
the structure of special $*$-simple complete ideals in terms of their Rees valuations. Let $T$ be an infinitely near point to $R$ with $\operatorname{dim} R=\operatorname{dim} T$ and $R / \mathbf{m}=T / \mathbf{m}_{T}$. We prove in Theorem 6.8 that the special $*$-simple complete ideal $P_{R T}$ has a unique Rees valuation if and only if either $\operatorname{dim} R=2$ or there is no change of direction in the unique finite sequence of local quadratic transformations from $R$ to $T$. In the case where $T=R_{1}$ is a first local quadratic transform of $R$ and $R / \mathbf{m} \neq T / \mathbf{m}_{T}$, we demonstrate in Examples 6.11 and 6.12 that sometimes the special $*$-simple complete ideal $P_{R T}$ has two Rees valuations and sometimes only one Rees valuation. Examples 6.13 and 6.14 illustrate a pattern where from $R_{0}$ to $R_{2}$ or from $R_{0}$ to $R_{3}$ there is exactly one or exactly two changes of direction.

\section{Preliminaries}

Let $V$ be a valuation domain and let $R$ be a subring of $V$. Let $\mathbf{m}(V)$ denote the unique maximal ideal of $V$. We call the prime ideal $\mathbf{m}(V) \cap R$ of $R$ the center of $V$ on $R$.

Let $(R, \mathbf{m})$ be a Noetherian local domain with field of fractions $\mathcal{Q}(R)$. A valuation domain $(V, \mathbf{m}(V))$ is said to birationally dominate $R$ if $R \subseteq V \subseteq \mathcal{Q}(R)$ and $\mathbf{m}(V) \cap R=\mathbf{m}$, that is, $\mathbf{m}$ is the center of $V$ on $R$. The valuation domain $V$ is said to be a prime divisor of $R$ if $V$ birationally dominates $R$ and the transcendence degree of the field $V / \mathbf{m}(V)$ over $R / \mathbf{m}$ is $\operatorname{dim} R-1$. If $V$ is a prime divisor of $R$, then $V$ is a DVR [A, p. 330].

The quadratic dilatation or blowup of $\mathbf{m}$ along $V$, cf. [N] page 141], is the unique local ring on the blowup $\mathrm{Bl}_{\mathbf{m}}(R)$ of $\mathbf{m}$ that is dominated by $V$. The ideal $\mathbf{m} V$ is principal and is generated by an element of $\mathbf{m}$. Let $a \in \mathbf{m}$ be such that $a V=\mathbf{m} V$. Then $R[\mathbf{m} / a] \subset V$. Let $Q:=\mathbf{m}(V) \cap R[\mathbf{m} / a]$. Then $R[\mathbf{m} / a]_{Q}$ is the quadratic transformation of $R$ along $V$. In the special case where $(R, \mathbf{m})$ is a $d$-dimensional regular local domain we use the following terminology.

Definition 2.1. Let $d$ be a positive integer and let $(R, \mathbf{m}, k)$ be a $d$-dimensional regular local ring with maximal ideal $\mathbf{m}$ and residue field $k$. Let $x \in \mathbf{m} \backslash \mathbf{m}^{2}$ and let $S_{1}:=R\left[\frac{\mathbf{m}}{x}\right]$. The ring $S_{1}$ is a $d$-dimensional regular ring in the sense that each localization of $S_{1}$ at a prime ideal is a regular local ring. To see this, observe that $S_{1} / x S_{1}$ is isomorphic to a polynomial ring in $d-1$ variables over the field $k$, cf. [SH, Corollary 5.5.9], and $S_{1}[1 / x]=R[1 / x]$ is a regular ring. Moreover, $S_{1}$ is a UFD since $x$ is a prime element of $S_{1}$ and $S_{1}[1 / x]=R[1 / x]$ is a UFD, cf. [M, Theorem 20.2]. 
Let $I$ is an $\mathbf{m}$-primary ideal of $R$ with $r:=\operatorname{ord}_{R}(I)$. Then one has in $S_{1}$

$$
I S_{1}=x^{r} I_{1} \quad \text { for some ideal } I_{1} \text { of } S_{1} .
$$

We observe in Remark 2.2 that either $I_{1}=S_{1}$ or ht $I_{1} \geq 2$. Thus $I_{1}$ is the transform $I^{S_{1}}$ of $I$ in $S_{1}$ as in Definiton 1.1 .

Let $\mathbf{p}$ be a prime ideal of $R\left[\frac{\mathbf{m}}{x}\right]$ with $\mathbf{m} \subseteq \mathbf{p}$. The local ring

$$
R_{1}:=R\left[\frac{\mathbf{m}}{x}\right]_{\mathbf{p}}=\left(S_{1}\right)_{\mathbf{p}}
$$

is called a local quadratic transform of $R$; the ideal $I_{1} R_{1}$ is the transform of $I$ in $R_{1}$ as in Definition 1.1 .

Remark 2.2. With the notation of Definition 2.1, to justify that the ideal $I_{1}$ is the transform of $I$ in $S_{1}$, we observe that the ideal $I_{1}$ is not contained in any heightone prime of $S_{1}$. For if $I_{1} \subseteq x S_{1}$, then we would have $I \subseteq x^{r+1} S_{1} \cap R=\mathbf{m}^{r+1}$, a contradiction to the choice of $r$. If $I_{1} \subseteq q$, where $q$ is a height-one prime of $S_{1}$ different from $x S_{1}$, then $I \subseteq q \cap R$. This is impossible since $q \cap R$ is a height-one prime of $R$ and $I$ is $\mathbf{m}$-primary.

We follow the notation of $[\mathrm{L}$ and refer to regular local rings of dimension at least two as points. A point $T$ is said to be infinitely near to a point $R$, in symbols, $R \prec T$, if there is a finite sequence of local quadratic transformations

$$
R=: R_{0} \subset R_{1} \subset R_{2} \subset \cdots \subset R_{n}=T \quad(n \geq 0),
$$

where $R_{i+1}$ is a local quadratic transform of $R_{i}$ for $i=0,1, \ldots, n-1$. If such a sequence of local quadratic transforms as in Equation 1 exists, then it is unique and it is called the quadratic sequence from $R$ to $T[\mathrm{~L}$, Definition 1.6].

Remark 2.3. Let $(R, \mathbf{m})$ be a regular local $\operatorname{ring}$ with $\operatorname{dim} R \geq 2$. As noted in [L, Proposition 1.7], there is a one-to-one correspondence between the points $T$ infinitely near to $R$ and the prime divisors $V$ of $R$. This correspondence is defined by associating with $T$ the order valuation $\operatorname{ring} V$ of $T$. Since $V$ is the unique local quadratic transform of $T$ of dimension one, the local quadratic sequence in Equation 1 extends to give Equation 2 .

$$
R=: R_{0} \subset R_{1} \subset R_{2} \subset \cdots \subset R_{n}=T \subset V .
$$

The one-to-one correspondence between the points $T$ infinitely near to $R$ and the prime divisors $V$ of $R$ implies that $T$ is the unique point infinitely near to $R$ for which the order valuation ring of $T$ is $V$. However, if $\operatorname{dim} R>2$, then there often 
exist regular local rings $S$ with $S \neq T$ such that $S$ birationally dominates $R$ and the order valuation ring of $S$ is $V$. We illustrate this in Example 2.4.

Example 2.4. Let $(R, \mathbf{m})$ be a 3 -dimensional RLR with $\mathbf{m}=(x, y, z) R$, and let $V$ denote the order valuation ring of $R$. Let $S=R\left[\frac{y}{x}\right]_{(x, z)}$. Then $S$ is a 2-dimensional RLR that birationally dominates $R$, and $V$ is the order valuation ring of $S$. Notice that $S$ is not infinitely near to $R$.

Remark 2.5. Let $(R, \mathbf{m})$ be a $d$-dimensional RLR with $d \geq 2$ and let $V$ be the order valuation ring of $R$. Let $(S, \mathbf{n})$ be a $d$-dimensional RLR that is a birational extension of $R$. Then

(1) $S$ dominates $R$.

(2) If $V$ dominates $S$, then $R=S$.

(3) Thus $R$ is the unique $d$-dimensional RLR having order valuation ring $V$ among the regular local rings birational over $R$.

Proof. For item (1), let $P:=\mathbf{n} \cap R$. Then $R_{P} \subseteq S$. If $P \neq \mathbf{m}$, then $\operatorname{dim} R_{P}=$ $n<d$. Since every birational extension of an $n$-dimensional Noetherian domain has dimension at most $n$, we must have $\operatorname{dim} S \leq n$, a contradiction. Thus $S$ dominates $R$. Item (2) follows from [Sa2, Corollary 2.6]. In more detail, if $V$ dominates $S$, then $R / \mathbf{m}=S / \mathbf{n}$ and the elements in a minimal generating set for $\mathbf{m}$ are part of a minimal generating set for $\mathbf{n}$. Hence we have $\mathbf{m} S=\mathbf{n}$. By Zariski's Main Theorem as in [N, (37.4)], it follows that $R=S$. Item (3) follows from item (2).

Example 2.6 demonstrates the existence of a prime divisor $V$ for a 3-dimensional $\operatorname{RLR}(R, \mathbf{m}, k)$ for which there exist infinitely many distinct 3-dimensional RLRs that birationally dominate $R$, and have $V$ as their order valuation ring.

Example 2.6. Let $(R, \mathbf{m}, k)$ be a 3-dimensional regular local ring with residue field $R / \mathbf{m}=k$ and maximal ideal $\mathbf{m}=(x, y, z) R$. Let $V$ be the prime divisor of $R$ corresponding to the valuation $v$, where $v(x)=v(y)=1$ and $v(z)=3$, and where the images of $\frac{x^{3}}{z}$ and $\frac{y^{3}}{z}$ in the residue field $k_{v}$ of $V$ are algebraically independent over $R / \mathbf{m}=k$. Then we have :

(1) In the unique finite sequence of local quadratic transformations given by $[\mathrm{L}$, Proposition 1.7], we have:

$$
R=: R_{0} \subset R_{1} \subset R_{2} \subset V,
$$


where

$$
\begin{aligned}
& R_{1}:=R\left[\frac{\mathbf{m}}{x}\right]_{\mathbf{p}}, \quad \text { where } \quad \mathbf{p}:=\mathbf{m}(V) \cap R\left[\frac{\mathbf{m}}{x}\right]=\left(x, \frac{z}{x}\right) R\left[\frac{\mathbf{m}}{x}\right], \\
& R_{2}:=R_{1}\left[\frac{\mathbf{m}_{1}}{x}\right]_{\mathbf{q}}, \quad \text { where } \quad \mathbf{q}:=\mathbf{m}(V) \cap R_{1}\left[\frac{\mathbf{m}_{1}}{x}\right]=\left(x, \frac{z}{x^{2}}\right) R_{1}\left[\frac{\mathbf{m}_{1}}{x}\right],
\end{aligned}
$$

and $V$ is the order valuation ring of $R_{2}$. Notice that $\left(R_{1}, \mathbf{m}_{1}\right)$ and $\left(R_{2}, \mathbf{m}_{2}\right)$ are 2-dimensional RLRs.

(2) For each integer $n \geq 1$, let $T_{n}:=R\left[\frac{z}{x^{2}+y^{2 n+1}}\right]_{\left(x, y, \frac{z}{x^{2}+y^{2 n+1}}\right)}$. Then we have:

(a) By [Sa1, Lemma 4.2], each $T_{n}$ is a 3 -dimensional RLR that birationally dominates $R$.

(b) For each $n$, the images of $\frac{y}{x}$ and $\frac{z}{x^{3}}$ in $k_{v}$ are algebraically independent over $k$. Hence the order valuation ring of $T_{n}$ is $V$.

(c) By [Sa1, Corollary 4.5]), the elements in the family $\left\{T_{n}\right\}_{n=1}^{\infty}$ are distinct.

Definition 2.7. A base point of a nonzero ideal $I \subset R$ is a point $T$ infinitely near to $R$ such that $I^{T} \neq T$. The set of base points of $I$ is denoted by

$$
\mathcal{B P}(I)=\left\{T \mid T \text { is a point such that } R \prec T \text { and } \operatorname{ord}_{T}\left(I^{T}\right) \neq 0\right\} .
$$

The point basis of a nonzero ideal $I \subset R$ is the family of nonnegative integers

$$
\mathcal{B}(I)=\left\{\operatorname{ord}_{T}\left(I^{T}\right) \mid R \prec T\right\} .
$$

The nonzero ideal $I$ is said to be finitely supported if $I$ has only finitely many base points.

Definition 2.8. Let $R \prec T$ be points such that $\operatorname{dim} R=\operatorname{dim} T$. Lipman proves in [L, Proposition 2.1] the existence of a unique complete ideal $P_{R T}$ in $R$ such that for every point $A$ with $R \prec A$, the complete transform

$$
\overline{\left(P_{R T}\right)^{A}} \text { is }\left\{\begin{array}{l}
\text { a } * \text {-simple ideal if } A \prec T, \\
\text { the ring } A \text { otherwise. }
\end{array}\right.
$$

The ideal $P_{R T}$ of $R$ is said to be a special $*$-simple complete ideal.

In the case where $R \prec T$ and $\operatorname{dim} R=\operatorname{dim} T$, we say that the order valuation ring of $T$ is a special prime divisor of $R$.

Remark 2.9. With notation at in Definition 2.8, a prime divisor $V$ of $R$ is special if and only if the unique point $T$ with $R \prec T$ such that the order valuation ring of $T$ is $V$ has $\operatorname{dim} T=\operatorname{dim} R$. Let $\operatorname{dim} R=d$. If $V$ is a special prime divisor of $R$, then the residue field of $V$ is a pure transcendental extension of degree $d-1$ of the residue field $T / \mathbf{m}(T)$ of $T$, and $T / \mathbf{m}(T)$ is a finite algebraic extension of 
$R / \mathbf{m}$. If the residue field $R / \mathbf{m}$ of $R$ is algebraically closed and $V$ is a special prime divisor of $R$, then the residue field of $V$ is a pure transcendental extension of $R / \mathbf{m}$ of transcendence degree $d-1$.

It would be interesting to identify and describe in other ways the special prime divisors of $R$ among the set of all prime divisors of $R$.

\section{FACtorization as PROduCtS OF SPECIAL $*$-Simple COMPLETE IDEALS}

Let $R=\alpha$ be a $d$-dimensional regular local ring with $d \geq 2$. Lipman in $[\mathrm{L}$, Theorem 2.5] proves that for every finitely supported complete ideal $I$ of $R$ there exists a unique family of integers

$$
\left(n_{\beta}\right)=\left(n_{\beta}(I)\right)_{\beta \succ \alpha, \operatorname{dim} \beta=\operatorname{dim} \alpha}
$$

such that $n_{\beta}=0$ for all but finitely many $\beta$ and such that

$$
\left(\prod_{n_{\delta}<0} P_{\alpha \delta}^{-n_{\delta}}\right) * I=\prod_{n_{\gamma}>0} P_{\alpha \gamma}^{n_{\gamma}}
$$

where $P_{\alpha \beta}$ is the special $*$-simple ideal associated with $\alpha \prec \beta$ and the products are $*$-products. The product on the left in Equation 3 is over all $\delta \succ \alpha$ such that $n_{\delta}<0$ and the product on the right is over all $\gamma \succ \alpha$ such that $n_{\gamma}>0$.

Lipman gives the following example to illustrate this decomposition.

Example 3.1. Let $k$ be a field and let $\alpha=R=k[[x, y, z]]$ be the formal power series ring in the 3 variables $x, y, z$ over $k$. Let

$$
\beta_{x}=R\left[\frac{y}{x}, \frac{z}{x}\right]_{(x, y / x, z / x)}, \quad \beta_{y}=R\left[\frac{x}{y}, \frac{z}{y}\right]_{(y, x / y, z / y)}, \quad \beta_{z}=R\left[\frac{x}{z}, \frac{y}{z}\right]_{(z, x / z, y / z)}
$$

be the local quadratic transformations of $R$ in the $x, y, z$ directions. The associated special $*$-simple ideals are

$$
P_{\alpha \beta_{x}}=\left(x^{2}, y, z\right) R, \quad P_{\alpha \beta_{y}}=\left(x, y^{2}, z\right) R, \quad P_{\alpha \beta_{z}}=\left(x, y, z^{2}\right) R .
$$

The equation

$$
(x, y, z)\left(x^{3}, y^{3}, z^{3}, x y, x z, y z\right)=P_{\alpha \beta_{x}} P_{\alpha \beta_{y}} P_{\alpha \beta_{z}}
$$

represents the factorization of the finitely supported ideal $I=\left(x^{3}, y^{3}, z^{3}, x y, x z, y z\right) R$ as a product of special $*$-simple ideals. Here $P_{\alpha \alpha}=(x, y, z) R$. The base points of $I$ are $\mathcal{B P}(I)=\left\{\alpha, \beta_{x}, \beta_{y}, \beta_{z}\right\}$ and the point basis of $I$ is $\mathcal{B}(I)=\{2,1,1,1\}$. Equation 4 represents the following equality of point bases

$$
\mathcal{B}\left(P_{\alpha \alpha}\right)+\mathcal{B}(I)=\mathcal{B}\left(P_{\alpha \beta_{x}}\right)+\mathcal{B}\left(P_{\alpha \beta_{y}}\right)+\mathcal{B}\left(P_{\alpha \beta_{z}}\right) .
$$


Each of $P_{\alpha \beta_{x}}, P_{\alpha \beta_{y}}, P_{\alpha \beta_{z}}$ has a unique Rees valuation. Their product has in addition the order valuation of $\alpha$ as a Rees valuation.

Question 3.2. Let $I$ be a finitely supported ideal of a regular local ring $R$.

(1) If the base points of $I$ are linearly ordered, does it follow that $I$ is a $*$ product of special $*$-simple complete ideals, i.e., in the factorization given in Equation 3 are all the integers $n_{\beta}$ nonnegative?

(2) If $I$ is $*$-simple and if the base points of $I$ are linearly ordered, does it follow that $I$ is a special $*$-simple ideal?

(3) If $R \prec T$ with $\operatorname{dim} R=\operatorname{dim} T$ and $R \neq T$, can it happen that some power of the special $*$-simple complete ideal $P_{R T}$ has the maximal ideal $\mathbf{m}$ of $R$ as a factor, that is, can there exist an ideal $Q$ of $R$ such that $\mathbf{m} Q=\left(P_{R T}\right)^{n}$ for some positive integer $n$ ? 1

\section{Rees valuations of ideals of a Regular local Ring}

We use the following setting.

Setting 4.1. Let $d$ be a positive integer and let $(R, \mathbf{m}, k)$ be a $d$-dimensional regular local ring with maximal ideal $\mathbf{m}$ and infinite residue field $k$. Let $I$ be an $\mathbf{m}$-primary ideal. For each $V \in$ Rees $I$, let $v$ denote the corresponding Rees valuation with value group $\mathbb{Z}$. Let $x \in \mathbf{m} \backslash \mathbf{m}^{2}$ be such that $x V=\mathbf{m} V$ for each $V \in$ Rees $I$. Since the field $k$ is infinite and the set Rees $I$ is finite, it is possible to choose such an element $x$. Let $r=\operatorname{ord}_{R} I$. As in Definition 2.1, we have $I S_{1}=x^{r} I_{1}$, where $I_{1}$ is the transform of $I$ in $S_{1}=R\left[\frac{\mathbf{m}}{x}\right]$.

Remark 4.2. With the notation of Setting 4.1, we have:

(1) If $J \subseteq I$ is a reduction of $I$ in $R$, then $\operatorname{ord}_{R} J=\operatorname{ord}_{R} I=r$, and $J S_{1}=x^{r} J_{1}$ is a reduction of $I S_{1}=x^{r} I_{1}$ in $S_{1}$. It follows that $J_{1}$ is the transform in $S_{1}$ of $J$ and $J_{1} \subseteq I_{1}$ is a reduction of $I_{1}$ in $S_{1}$.

(2) If $J=\left(a_{1}, \ldots, a_{d}\right) R$ is a reduction of $I$ then a DVR $V$ that birationally dominates $R$ is a Rees valuation ring of $I$ if and only if the images of $\frac{a_{2}}{a_{1}}, \ldots, \frac{a_{d}}{a_{1}}$ in the field $\frac{V}{\mathbf{m}(V)}$ are algebraically independent over $k$.

(3) The unique Rees valuation ring of $\mathbf{m}$ is $\left(S_{1}\right)_{x S_{1}}$, i.e., Rees $\mathbf{m}=\left\{\left(S_{1}\right)_{x S_{1}}\right\}$.

\footnotetext{
${ }^{1}$ In a joint paper with Matthew Toeniskoetter titled "Finitely supported $*$-simple complete ideals in a regular local ring", we have answered these three questions in the case where $I$ is a finitely supported monomial ideal.
} 
Proposition 4.3. With the notation of Setting 4.1, we have:

(1) If $I_{1}=S_{1}$, then $v=\operatorname{ord}_{R}$ and $\operatorname{ord}_{R}$ is the unique Rees valuation of $I$.

(2) If $I_{1} \neq S_{1}$ and $v \neq \operatorname{ord}_{R}$, then $V \in \operatorname{Rees}_{S_{1}} I_{1}$.

(3) In general, we have Rees $I \subseteq \operatorname{Rees}_{S_{1}} I_{1} \cup$ Rees $\mathbf{m}$.

Proof. For the proof of item (1), let $\mathbf{p}:=\mathbf{m}(V) \cap S_{1}$ be the center of $V$ on $S_{1}$. Since $x S_{1}=\mathbf{m} S_{1} \subseteq \mathbf{p}$ and $\left(S_{1}\right)_{x S_{1}}$ is the valuation ring of $\operatorname{ord}_{R}$, it suffices to show that ht $\mathbf{p}=1$. By the Dimension Formula ([M], page 119]), we have

$$
\text { ht } \mathbf{p}=1 \Longleftrightarrow \operatorname{tr} \cdot \operatorname{deg}_{k} \kappa\left(S_{1} / \mathbf{p}\right)=d-1,
$$

where $\kappa\left(S_{1} / \mathbf{p}\right)$ denotes the field of fractions of $S_{1} / \mathbf{p}$. Let $J:=\left(a_{1}, \ldots, a_{d}\right) R$ be a reduction of $I$. Since $V \in \operatorname{Rees} I=\operatorname{Rees} J$, the images of $a_{2} / a_{1}, \ldots, a_{d} / a_{1}$ in $V / \mathbf{m}(V)=: k_{v}$ are algebraically independent over $k$. Since $J S_{1}$ is a reduction of $I S_{1}$ and $I S_{1}=x^{r} S_{1}$ is a principal ideal, we have

$$
J_{1}:=\left(f_{1}, \ldots, f_{d}\right) S_{1}=S_{1}, \quad \text { where } \quad f_{i}:=\frac{a_{i}}{x^{r}} \quad \text { for } \quad 1 \leq i \leq d .
$$

It follows that $\left(f_{1}, \ldots, f_{d}\right) V=V$ and thus $v\left(f_{i}\right)=0$ for $i=1, \ldots, d$. Consider the inclusion maps:

$$
\frac{S_{1}}{\mathbf{p}} \hookrightarrow \kappa\left(S_{1} / \mathbf{p}\right) \hookrightarrow \frac{V}{\mathbf{m}(V)}
$$

Since $v\left(f_{i}\right)=0$ and $\mathbf{p}=\mathbf{m}(V) \cap S_{1}$, we have $f_{i} \in S_{1} \backslash \mathbf{p}$. Therefore the images of $\frac{f_{2}}{f_{1}}=\frac{a_{2}}{a_{1}}, \ldots, \frac{f_{d}}{f_{1}}=\frac{a_{d}}{a_{1}}$ in $\frac{V}{\mathbf{m}(V)}$ are in the subfield $\kappa\left(S_{1} / \mathbf{p}\right)$ of $\frac{V}{\mathbf{m}(V)}$. Hence $\operatorname{tr} . \operatorname{deg}_{k} \kappa\left(S_{1} / \mathbf{p}\right)=d-1$.

For the proof of item (2), we use the notation of the proof of item (1). Notice that $f_{1}, \ldots, f_{d}$ all have the same $v$-value. Moreover, since $V \neq\left(S_{1}\right)_{x S_{1}}$, we must have $v\left(f_{i}\right)>0$; for if $v\left(f_{i}\right)=0$, the proof of item (1) shows that ht $\mathbf{p}=1$ and thus $V=\left(S_{1}\right)_{x S_{1}}$, a contradiction to our assumption. Thus $J_{1}=\left(f_{1}, \ldots, f_{d}\right) S_{1} \subseteq \mathbf{p}$. Since $J_{1}$ is a reduction of $I_{1}$ and the images of $\frac{f_{2}}{f_{1}}=\frac{a_{2}}{a_{1}}, \ldots, \frac{f_{d}}{f_{1}}=\frac{a_{d}}{a_{1}}$ in $\frac{V}{\mathbf{m}(V)}$ are algebraically independent over $k$, and thus we have $V \in \operatorname{Rees}_{S_{1}} J_{1}=\operatorname{Rees}_{S_{1}} I_{1}$.

Item (3) follows from items (1) and (2).

Proposition 4.4. Let the notation be as in Setting 4.1 and let $V \in$ Rees $I$. As in Equation 2, there exists a unique finite sequence of local quadratic transforms

$$
R=: R_{0} \subset R_{1} \subset R_{2} \subset \cdots \subset R_{n}=T \subset V,
$$

where $V$ is the order valuation ring of $T$. Then the points $R_{0}, \ldots, R_{n}$ are all base points of $I$. 
Proof. If $V$ is the order valuation ring of $R$, then $n=0$ in Equation 5 and $R_{0}$ is a base point of $I$. If $n>0$, consider $S_{1}=R\left[\frac{\mathbf{m}}{x}\right]$ as in Setting 4.1, By Proposition 4.3, $V \in \operatorname{Rees}_{S_{1}} I_{1}$. The local quadratic transform $R_{1}$ of $R$ is a localization of $S_{1}$ and $I_{1} R_{1}$ is a proper ideal in $R_{1}$. By an inductive argument on the length $n$ of the sequence in Equation 5, we conclude that the points $R_{1}, \ldots, R_{n}$ are base points of $I_{1} R_{1}$ and therefore also base points of $I$.

Remark 4.5. Let the notation be as in Setting 4.1. If $I$ is a finitely supported ideal in $R$, then [L, Corollary 1.22] implies that ht $I_{1}=d$ and $\operatorname{dim}\left(S_{1} / I_{1}\right)=0$.

Proposition 4.6. Let the notation be as in Setting 4.1. If I is a finitely supported ideal in $R$, then

$$
\operatorname{Rees}_{S_{1}} I_{1} \subseteq \text { Rees } I \text {. }
$$

Proof. By Proposition 4.3, we have $I_{1}=S_{1}$ if and only if $\operatorname{ord}_{R}$ is the unique Rees valuation of $I$. Assume $I_{1} \neq S_{1}$, and let $J:=\left(a_{1}, \ldots, a_{d}\right) R$ be a reduction of $I$. Since $J S_{1}$ is a reduction of $I S_{1}$ and $I_{1} \neq S_{1}$, we have

$$
J_{1}:=\left(f_{1}, \ldots, f_{d}\right) S_{1} \neq S_{1}, \quad \text { where } \quad f_{i}:=\frac{a_{i}}{x^{r}} \quad \text { for } \quad 1 \leq i \leq d .
$$

It follows that $J_{1}$ is a reduction of $I_{1}$. By Remark 4.5, we have ht $J_{1}=d$. Hence $\left\{f_{1}, \ldots, f_{d}\right\}$ is a minimal set of generators of $J_{1}$. For each $W \in \operatorname{Rees}_{S_{1}} I_{1}=$ $\operatorname{Rees}_{S_{1}} J_{1}$, the images of $f_{2} / f_{1}, \ldots, f_{d} / f_{1}$ in $W / \mathbf{m}(W)$ are algebraically independent over $k$. Let $\mathfrak{q}:=\mathbf{m}(W) \cap \overline{R\left[\frac{J}{a_{1}}\right]}$ be the center of $W$ on $A:=\overline{R\left[\frac{J}{a_{1}}\right]}$. Since $\frac{f_{2}}{f_{1}}=\frac{a_{2}}{a_{1}}, \ldots, \frac{f_{d}}{f_{1}}=\frac{a_{d}}{a_{1}}$, we have that the images of $\frac{a_{2}}{a_{1}}, \ldots, \frac{a_{d}}{a_{1}}$ in $\frac{W}{\mathbf{m}(W)}$ are in the subfield $\kappa(A / \mathfrak{q})$ of $\frac{W}{\mathbf{m}(W)}$. Hence tr. $\operatorname{deg}_{k} \kappa(A / \mathfrak{q})=d-1$. By the Dimension Formula $\left(\left[\mathrm{M}\right.\right.$, page 119]), ht $(\mathfrak{q})=1$. Hence $A_{\mathfrak{q}}=W$.

Propositions 4.3 and 4.6 imply the following corollary.

Corollary 4.7. Let the notation be as in Setting 4.1. If I is a finitely supported ideal in $R$ and $\operatorname{ord}_{R}$ is not a Rees valuation of $I$, then

$$
\operatorname{Rees}_{S_{1}} I_{1}=\text { Rees } I \text {. }
$$

We use Proposition 4.8 to demonstrate that without the assumption in Proposition 4.6 that the ideal $I$ has finite support, there sometimes exist Rees valuations of the transform $I_{1}$ of $I$ that are not Rees valuations of $I$. 
Proposition 4.8. With the notation of Setting 4.1, if each Rees valuation of I is centered on a maximal ideal of $S_{1}$ and $\operatorname{dim}\left(S_{1} / I_{1}\right)>0$, then there exist Rees valuations of $I_{1}$ that are not Rees valuations of $I$, i.e., Rees $S_{1} I_{1} \nsubseteq$ Rees $I$.

Proof. Since $\operatorname{dim}\left(S_{1} / I_{1}\right)>0$, there exists a minimal prime $P$ of $I_{1}$ such that $P$ is not a maximal ideal of $S_{1}$. Every minimal prime of $I_{1}$ is the center of at least one Rees valuation ring of $I_{1}$. Let $V \in \operatorname{Rees}_{S_{1}} I_{1}$ be centered on $P$. By assumption, $V \notin$ Rees $I$.

We present in Example 4.9 a specific example where the hypotheses of Proposition 4.8 hold. By Proposition 4.6, the ideal $I$ of Example4.9 is not finitely supported.

Example 4.9. Let $(R, \mathbf{m}, k)$ be a three-dimensional regular local ring with residue field $R / \mathbf{m}=k$ and maximal ideal $\mathbf{m}=(x, y, z) R$. Let

$$
J:=\left(x^{2}, y^{3}, z^{5}\right) R, \quad \text { and } \quad S_{1}:=R\left[\frac{\mathbf{m}}{z}\right] \quad \text { with } \quad x_{1}:=\frac{x}{z} \quad y_{1}:=\frac{y}{z} .
$$

The ideal $I:=\left(x^{2}, y^{3}, z^{5}, x y^{2}, x y z^{2}, y^{2} z^{2}, y z^{4}\right) R$ is the integral closure of $J$, and :

(1) The ideals $J$ and $I$ have a unique Rees valuaton $v$, where

$$
v(x)=15, \quad v(y)=10, \quad \text { and } \quad v(z)=6,
$$

and the images of $\frac{x^{2}}{z^{5}}$ and $\frac{y^{3}}{z^{5}}$ in $k_{v}$ are algebrically independent over $k$.

(2) The center of $v$ on $S_{1}$ is the maximal ideal $\left(x_{1}, y_{1}, z\right) S_{1}$.

(3) $J_{1}=\left(x_{1}^{2}, z y_{1}^{3}, z^{3}\right) S_{1} \subset I_{1}=\left(x_{1}^{2}, z y_{1}^{3}, z^{3}, x_{1} y_{1}^{2} z, x_{1} y_{1} z^{2}, y_{1}^{2} z^{2}\right) S_{1}$. We have $J_{1}$ is a reduction of $I_{1}$ with ht $J_{1}=2$ and $\mu\left(J_{1}\right)=3$.

(4) The ideal $I$ is not finitely supported.

(5) $\operatorname{Rees}_{S_{1}} I_{1}=\{V, W\}$, where $V$ and $W$ denote the valuation rings corresponding to $v$ and $w$, respectively, and where

$$
w(x)=3, \quad w(y)=2, \quad \text { and } \quad w(z)=2,
$$

and the images of $\frac{x^{2}}{y^{3}}$ and $\frac{x^{2}}{z^{3}}$ in $k_{w}$ are algebrically independent over $k$.

(6) $\operatorname{Rees}_{S_{1}} I_{1} \nsubseteq$ Rees $I$.

Proof. The assertion in item (1) is well-known, see for example [SH, page 209], and item (2) follows from item (1). Since $I_{1} \subseteq\left(x_{1}, z\right) S_{1}$, we have ht $I_{1}=2$ as asserted in item (3). Item (4) follows from Remark 4.5. For the proof of item (5), since $v$ is not $\operatorname{ord}_{R}$, Proposition 4.3 implies that $V \in \operatorname{Rees}_{S_{1}} I_{1}$. We have $I_{1} \subseteq \mathfrak{p}:=\left(x_{1}, z\right) S_{1}$. Moreover : 
(1) $y_{1}$ is a unit in the two-dimensional regular local ring $\left(S_{1}\right)_{\mathfrak{p}}$. Also $\mathfrak{p} \cap R=\mathbf{m}$ and the image of $y_{1}$ in the field of fractions of $S_{1} / \mathfrak{p}$ is algebrically independent over $R / \mathbf{m}$.

(2) $\left(I_{1}\right)_{\mathfrak{p}}=\left(x_{1}^{2}, z\right)$ is a simple complete ideal in $\left(S_{1}\right)_{\mathfrak{p}}$.

(3) Rees $\left(I_{1}\right)_{\mathfrak{p}}=\{W\}$, where $w\left(x_{1}\right)=1, w(z)=2$, and the image of $\frac{x_{1}^{2}}{z}$ in $k_{w}$ is algebraically independent over the field of fractions of $S_{1} / \mathfrak{p}$.

(4) $\operatorname{Rees}_{S_{1}}\left(I_{1}\right)=\{V, W\}$, where $w(x)=3, w(y)=z$, and $w(z)=2$.

In Example 4.10, the height of the transform ideal $I_{1}$ is less than the height of $I$ and yet Rees $I=\operatorname{Rees}_{S_{1}} I_{1}$.

Example 4.10. Let $(R, \mathbf{m}, k)$ be a three-dimensional regular local ring with residue field $R / \mathbf{m}=k$ and and maximal ideal $\mathbf{m}=(x, y, z) R$. Let

$$
J:=\left(x^{2}, y^{2}, z\right) R, \quad \text { and } \quad S_{1}:=R\left[\frac{\mathbf{m}}{x}\right] \quad \text { with } \quad y_{1}:=\frac{y}{x} \quad z_{1}:=\frac{z}{x} .
$$

The ideal $I:=\left(x^{2}, x y, y^{2}, z\right) R$ is the integral closure of $J$, and :

(1) The ideals $J$ and $I$ have a unique Rees valuation $v$, where

$$
v(x)=1, \quad v(y)=1, \quad \text { and } \quad v(z)=2,
$$

and the images of $\frac{x^{2}}{z}$ and $\frac{y^{2}}{z}$ in $k_{v}$ are algebraically independent over $k$.

(2) $J_{1}=I_{1}=\left(x, z_{1}\right) S_{1}$, and hence ht $I_{1}=2$ and $\mu\left(I_{1}\right)=2$.

(3) $I$ is not finitely supported.

(4) $\operatorname{Rees}_{S_{1}} I_{1}=$ Rees $I$.

Proof. Item (1) is well-known, cf. [SH, Theorem 10.3.5]. Item (2) is clear and item (3) follows from Remark 4.5, For the proof of item (4), since $\mathfrak{p}:=I_{1}$ is a height two prime in $S_{1}$. Then :

(1) $y_{1}$ is unit in a two-dimensional regular local $\operatorname{ring}\left(S_{1}\right)_{\mathfrak{p}}$. Also $\mathfrak{p} \cap R=\mathbf{m}$ and the image of $y_{1}$ in the field of fractions of $S_{1} / \mathfrak{p}$ is algebraically independent over $R / \mathbf{m}$.

(2) $\operatorname{ord}_{\left(S_{1}\right)_{\mathfrak{p}}}$ is the unique Rees valuation of $\mathfrak{p}$. To see that this valuation is $v$, observe that $v(x)=v\left(z_{1}\right)=1$ and the image of $\frac{z_{1}}{x}$ in $k_{v}$ is algebraically independent over the subfield $\left(S_{1}\right)_{\mathfrak{p}} / \mathfrak{p}\left(S_{1}\right)_{\mathfrak{p}}$ of $k_{v}$. This follows because the images of $y_{1}$ and $\frac{z_{1}}{x}$ in $k_{v}$ are algebraically independent over $k$. 


\section{Projectively full finitely supported complete ideals}

We use the following definitions:

Definition 5.1. Let $I$ be a regular proper ideal in a Noetherian ring $R$.

(1) An ideal $J$ in $R$ is projectively equivalent to $I$, if some powers of $I$ and $J$ have the same integral closure, i.e., $\overline{I^{j}}=\overline{J^{i}}$ for some $i, j \in \mathbb{Z}^{+}$.

(2) The ideal $I$ is said to be projective full, if the only complete ideals that are projectively equivalent to $I$ are the ideals $\overline{I^{k}}$ with $k \in \mathbb{Z}^{+}$.

The concept of projective equivalence of ideals was introduced by Samuel in Sam and further developed by Nagata in Nag. Making use of work of Rees in Rees, McAdam, Ratliff, and Sally in [MRS, Corollary 2.4] prove that the set $\mathcal{P}(I)$ of complete ideals projectively equivalent to $I$ is linearly ordered by inclusion and discrete. Moreover, if $I$ and $J$ are projectively equivalent, then Rees $I=\operatorname{Rees} J$ and the values of $I$ and $J$ with respect to these Rees valuation rings are proportional [MRS, Proposition 2.10]. If there exists a projectively full ideal $J$ that is projectively equivalent to $I$, then the set $\mathcal{P}(I)$ is said to be projectively full. As described in [CHRR1, there is naturally associated to the projective equivalence class of $I$ a numerical semigroup $\mathcal{S}(I)$. One has $\mathcal{S}(I)=\mathbb{N}$, the semigroup of nonnegative integers under addition, if and only if $\mathcal{P}(I)$ is projectively full.

Remark 5.2. Let $I$ be a finitely supported complete ideal of a $d$-dimensional regular local ring $R$, where $d \geq 2$.

(1) Every ideal projectively equivalent to $I$ is finitely supported.

(2) If an ideal $J$ is projectively equivalent to $I$, then the point bases $\mathcal{B}(I)$ and $\mathcal{B}(J)$ are proportional; indeed, if $\overline{I^{n}}=\overline{J^{m}}$ for positive integers $n$ and $m$, then $n \mathcal{B}(I)=m \mathcal{B}(J)$. In particular, if $I$ and $J$ are projectively equivalent, then $I$ and $J$ have the same base points.

(3) If the greatest common divisor (GCD) of the entries in $\mathcal{B}(I)$ is 1 , then the ideal $I$ is projectively full.

(4) Every special $*$-simple complete ideal is projectively full.

Proof. These statements all follow from [L, Remark 1.9 and Proposition 1.10]. We write out the details for item $(3)$. Let $\mathcal{B P}(I)=\left\{R_{0}, R_{1}, \ldots, R_{s}\right\}$, where $R_{0}:=R$ and $\mathcal{B}(I)=\left\{\operatorname{ord}_{R_{i}}\left(I^{R_{i}}\right)\right\}_{i=0}^{s}$. Let $J$ be a complete ideal that is projectively equivalent to $I$. Then $\overline{I^{n}}=\overline{J^{m}}$ for some $n, m \in \mathbb{Z}^{+}$. By [L, Proposition 1.10], we have 
$n \mathcal{B}(I)=\mathcal{B}\left(I^{n}\right)=\mathcal{B}\left(J^{m}\right)=m \mathcal{B}(J)$. Let $a_{i}:=\operatorname{ord}_{R_{i}}\left(I^{R_{i}}\right)$ for $i=0, \ldots, s$. Then we have

$$
n \mathcal{B}(I)=n\left\{a_{0}, a_{1}, \ldots, a_{s}\right\}=m\left\{b_{0}, b_{1}, \ldots, b_{s}\right\}=m \mathcal{B}(J),
$$

where $b_{i}=\operatorname{ord}_{R_{i}}\left(J^{R_{i}}\right)$ for $i=0,1, \ldots, s$. Since $G C D\left\{a_{0}, a_{1}, \ldots, a_{s}\right\}=1$, we have

$n=n\left(G C D\left\{a_{0}, a_{1}, \ldots, a_{s}\right\}\right)=G C D\left\{n a_{0}, n a_{1}, \ldots, n a_{s}\right\}=m\left(G C D\left\{b_{0}, b_{1}, \ldots, b_{s}\right\}\right)$.

Hence $n=m r$, where $r=G C D\left\{b_{0}, b_{1}, \ldots, b_{s}\right\}$, and therefore applying [L, Remark 1.9 and Proposition 1.10], we have

$$
\begin{aligned}
\overline{I^{m r}}=\overline{J^{m}} & \Longleftrightarrow \mathcal{B}\left(I^{m r}\right)=\mathcal{B}\left(J^{m}\right) \\
& \Longleftrightarrow m r \mathcal{B}(I)=m \mathcal{B}(J) \\
& \Longleftrightarrow r \mathcal{B}(I)=\mathcal{B}(J) \\
& \Longleftrightarrow \mathcal{B}\left(I^{r}\right)=\mathcal{B}(J) \\
& \Longleftrightarrow \overline{I^{r}}=\bar{J} \\
& \Longleftrightarrow \overline{I^{r}}=J, \quad \text { since } J \text { is complete. }
\end{aligned}
$$

Thus $I$ is projective full. Item (4) is immediate from item (3), because the last nonzero entry in the point basis of a special $*$-simple complete ideal is 1 .

Remark 5.3. Let $(\alpha=R, \mathbf{m})$ be a $d$-dimensional regular local ring with $d \geq 2$, and let $I$ be a complete finitely supported $\mathbf{m}$-primary ideal. Let $\mathcal{B}(I)=\left\{a_{0}, a_{1}, \ldots, a_{s}\right\}$ be the point basis of $I$. By Lipman's unique factorization thoerem: there exists a unique factorization as in Equation 3

$$
\left(\prod_{n_{\delta}<0} P_{\alpha \delta}^{-n_{\delta}}\right) * I=\prod_{n_{\gamma}>0} P_{\alpha \gamma}^{n_{\gamma}}
$$

If $d:=G C D\left\{a_{0}, a_{1}, \ldots, a_{s}\right\}$, the proof of this unique factorization implies that each exponent $n_{\delta}$ and $n_{\gamma}$ is a multiple of $d$. In particular, if there are no negative exponents in this factorization, then there exists an ideal $K$ such that $\overline{K^{d}}=I$.

In the two-dimensional case, the Zariski unique factorization theorem implies that $\mathcal{P}(I)$ is projectively full, and $I$ is projectively full if and only if the GCD of the entries in the point basis of $I$ is equal to 1 .

In the higher dimensional case, we ask:

Question 5.4. Let $I$ be a finitely supported complete ideal in a $d$-dimensional RLR.

(1) If $I$ is projectively full, does it follow that the GCD of the entries in the point basis of $I$ is equal to 1 ? 
(2) If $I$ is $*$-simple, is $I$ projectively full?

(3) Is $\mathcal{P}(I)$ always projectively full?

Remark 5.5. With the notation as in Setting 4.1, it is possible that $I$ is projectively full in $R$, while the transform $I_{1}$ is not projectively full in $S_{1}$. For example, let $d=2$ and $\mathbf{m}=(x, y) R$, and let

$$
I=\left(x^{2}, y\right)^{2} \mathbf{m}=\left(x^{5}, x^{3} y, x y^{2}, y^{3}\right) R .
$$

Since $\mathbf{m}$ is a simple factor of $I$, the ideal $I$ is projectively full in $R$, cf. [CHRR2, Example 3.2]. We have $S_{1}=R\left[\frac{y}{x}\right]$. Let $y_{1}=\frac{y}{x}$. Then $I S_{1}=x^{3} I_{1}$, where $I_{1}=$ $\left(x^{2}, x y_{1}, y_{1}^{2}\right) S_{1}$. Thus the ideal $I_{1}=\left(x, y_{1}\right)^{2} S_{1}$ is not projectively full.

Proposition 5.6. Let I be a complete $\mathbf{m}$-primary ideal of a regular local ring $(R, \mathbf{m})$ of dimension $d \geq 2$. With the notation as in Setting 4.1, if the transform $I_{1}$ of I in $S_{1}$ is projectively full, then $I$ is projectively full in $R$.

Proof. Let $J$ be an ideal in $R$ that is projectively equivalent to $I$, say $\overline{I^{n}}=\overline{J^{m}}$ with $n, m$ positive integers. Assume that $r=\operatorname{ord}_{R} I$ and $s=\operatorname{ord}_{R} J$. Then $I S_{1}=x^{r} I_{1}$ and $J S_{1}=x^{s} J_{1}$. Thus taking complete transforms, we have

$$
x^{r n} \overline{I_{1}^{n}}=\overline{I^{n} S_{1}}=\overline{J^{m} S_{1}}=x^{s m} \overline{J_{1}^{m}} .
$$

Since neither of the ideals $I_{1}$ nor $J_{1}$ in the UFD $S_{1}$ is contained in a proper principal ideal of $S_{1}$, we have $r n=s m$ and $\overline{I_{1}^{n}}=\overline{J_{1}^{m}}$. Thus $I_{1}$ and $J_{1}$ are projectively equivalent. Since $I_{1}$ is projectively full, $n=m t$ for some positive integer $t$. It follows that $\overline{I^{t}}=\bar{J}$.

\section{The STRUCTURE OF SPECIAL $*$-SIMPLE COMPLETE IDEALS}

Setting 6.1. We consider the structure of special $*$-simple complete ideals as in Definition 2.8. In the case where $\operatorname{dim} R=2$ and $R \prec T$, the special $*$-simple complete ideal $P_{R T}$ has a unique Rees valuation $\operatorname{ord}_{T}$. In the higher dimensional case, the ideal $P_{R T}$ has $\operatorname{ord}_{T}$ as a Rees valuation and often also has other Rees valuations. We observe in Proposition 6.4 that the other Rees valuations of $P_{R T}$ are in the set $\left\{\operatorname{ord}_{R_{i}}\right\}_{i=0}^{n-1}$, where

$$
R=: R_{0} \subset R_{1} \subset R_{2} \subset \cdots \subset R_{n}=T \quad(n \geq 2),
$$

where $R_{i+1}$ is a local quadratic transform of $R_{i}$ for $i=0,1, \ldots, n-1$, and $\operatorname{dim} R=$ $\operatorname{dim} T$. The residue field $R_{n} / \mathbf{m}_{n}$ of $R_{n}$ is a finite algebraic extension of the residue 
field $R_{0} / \mathbf{m}_{0}$ of $R_{0}$. If $R_{0} / \mathbf{m}_{0}=R_{n} / \mathbf{m}_{n}$, we observe in Corollary 6.6 that the other Rees valuations of $P_{R T}$ are in the set $\left\{\operatorname{ord}_{R_{i}}\right\}_{i=0}^{n-2}$.

Definition 6.2. We say there is no change of direction for the local quadratic sequence $R_{0}$ to $R_{n}$ in Equation [6] if there exists an element $x \in \mathbf{m}_{0}$ that is part of a minimal generating set of $\mathbf{m}_{n}$. We say there is a change of direction between $R_{0}$ and $R_{n}$ if $\mathbf{m}_{0} \subseteq \mathbf{m}_{n}^{2}$.

Remark 6.3. With notation as in Setting 6.1, assume that $\operatorname{dim} R=\operatorname{dim} T$, and let $I=P_{R_{0} R_{n}}$.

(1) By [L, Corollary 2.2], the transform $I^{R_{j}}=P_{R_{j} R_{n}}$ for all $j$ with $0 \leq j \leq n$. By Proposition 4.6, we have $\operatorname{Rees}_{R_{j}} I^{R_{j}} \subseteq \operatorname{Rees} I$. Thus for each $j$ with $0 \leq j \leq n$, we have

$$
\operatorname{Rees}_{R_{j}} P_{R_{j} R_{n}}=\operatorname{Rees}_{R_{j}} I^{R_{j}} \subseteq \text { Rees } I,
$$

and the number of Rees valuations of $I$ is greater than or equal to the number of Rees valuations of $P_{R_{j} R n}$.

(2) If $R_{0} / \mathbf{m}_{0}=R_{n} / \mathbf{m}_{n}$, then there is no change of direction in the local quadratic sequence from $R_{0}$ to $R_{n} \Longleftrightarrow \operatorname{ord}_{R_{0}}(I)=1 \quad \Longleftrightarrow \quad \mathcal{B}(I)=$ $\{1,1, \ldots, 1,1\}$.

Proposition 6.4. Let $(R, \mathbf{m}, k)$ be d-dimensional regular local ring, where $d \geq 2$, and let $R \prec T$ with $\operatorname{dim} T=d$. Assume the sequence of local quadratic transforms from $R$ to $T$ is as in Equation [6. Let $P_{R_{0} R_{n}}$ be the associated special *-simple complete $\mathbf{m}$-primary ideal in $R$, and let $V_{i}$ denote the valuation ring of $\operatorname{ord}_{R_{i}}$ for $0 \leq i \leq n$. Then we have

$$
\left\{V_{n}\right\} \subseteq \text { Rees } P_{R_{0} R_{n}} \subseteq\left\{V_{0}, V_{1}, \ldots, V_{n-1}, V_{n}\right\}
$$

Proof. Let $I:=P_{R_{0} R_{n}}$. Since $I^{R_{n}}=P_{R_{n} R_{n}}$ is the maximal ideal of $R_{n}$, we have $\left\{V_{n}\right\} \subseteq$ Rees $P_{R_{0} R_{n}}$. Let $V \in \operatorname{Rees} I$. We use the notation of Setting 4.1. Then $I S_{1}=x^{r} I_{1}$, where $r:=\operatorname{ord}_{R}(I)$. By [L, Corollary (2.2)], $I$ is a finitely supported ideal in $R$ and

$$
\mathcal{B P}(I)=\left\{R_{0}, R_{1}, \ldots, R_{n}\right\}
$$

Hence $R_{1}$ is the only base point of $I$ in the first neighborhood of $R$, and $I_{1}$ is contained in a unique maximal ideal $N_{1}$ in $S_{1}$. Hence $R_{1}=\left(S_{1}\right)_{N_{1}}$ and $\mathbf{m}_{1}:=N_{1} R_{1}$. 
By Proposition 4.3, we have

$$
\text { Rees } \begin{aligned}
P_{R_{0} R_{n}}=\text { Rees } I & \subseteq \operatorname{Rees}_{S_{1}} I_{1} \cup \text { Rees } \mathbf{m} \\
& =\operatorname{Rees}_{R_{1}} I_{1} R_{1} \cup \text { Rees } \mathbf{m} \\
& =\operatorname{Rees}_{R_{1}} P_{R_{1} R_{n}} \cup \operatorname{Rees} \mathbf{m} .
\end{aligned}
$$

Let $\mathbf{m}_{i}$ denote the maximal ideal of $R_{i}$ for $i=1, \ldots, n$. Since $I^{R_{1}}=P_{R_{1} R_{n}}$, a simple induction argument proves that

$$
\begin{aligned}
\text { Rees } P_{R_{0} R_{n}} & \subseteq \operatorname{Rees}_{R_{1}} P_{R_{1} R_{n}} \cup \operatorname{Rees} \mathbf{m} \\
& \subseteq \operatorname{Rees}_{R_{2}} P_{R_{2} R_{n}} \cup \operatorname{Rees}_{R_{1}} \mathbf{m}_{1} \cup \operatorname{Rees} \mathbf{m} \\
& \subseteq \cdots \\
& \subseteq \operatorname{Rees}_{R_{n}} \mathbf{m}_{n} \cup \operatorname{Rees}_{R_{n-1}} \mathbf{m}_{n-1} \cup \operatorname{Rees}_{R_{n-2}} \mathbf{m}_{n-2} \cup \cdots \cup \operatorname{Rees}_{R_{1}} \mathbf{m}_{1} \cup \operatorname{Rees} \mathbf{m} .
\end{aligned}
$$

We describe in Remark 6.5 the structure of a special *-simple complete ideal $P_{R_{0} R_{1}}$ in the case where $R_{1} / \mathbf{m}_{1}=R_{0} / \mathbf{m}_{0}$. This case always occurs if $R_{0} / \mathbf{m}_{0}$ is algebraically closed.

Remark 6.5. Let $R=R_{0}$ be a $d$-dimensional regular local ring and let $R_{1}$ be a local quadratic transform of $R$ with $\operatorname{dim} R_{1}=d$. Let $P_{R_{0} R_{1}}$ be the associated special *-simple complete ideal of $R_{0}$. With notation as in Setting 4.1, we may assume that

$$
S_{1}=R_{0}\left[\frac{\mathbf{m}_{0}}{x_{1}}\right]=R_{0}\left[\frac{x_{2}}{x_{1}}, \ldots, \frac{x_{d}}{x_{1}}\right] \subset R_{1},
$$

where $\mathbf{m}_{0}:=\mathbf{m}=\left(x_{1}, \ldots, x_{d}\right) R_{0}$. Then $R_{1}=\left(S_{1}\right)_{N_{1}}$, where $N_{1}$ is a maximal ideal in $S_{1}$ containing $x_{1} S_{1}=\mathbf{m} S_{1}$. Assume that $R_{1} / \mathbf{m}_{1}=R_{0} / \mathbf{m}_{0}$.

(1) Then $N_{1}=\left(x_{1}, \frac{x_{2}}{x_{1}}-a_{2}, \ldots, \frac{x_{d}}{x_{1}}-a_{d}\right) S_{1}$, where $a_{2}, \ldots, a_{d} \in R_{0}$. We have

$$
P_{R_{0} R_{1}}=\left(x_{1}^{2}, x_{2}-a_{2} x_{1}, \ldots, x_{d}-a_{d} x_{1}\right) R_{0},
$$

and the ideal $P_{R_{0} R_{1}}$ has unique Rees valuation $w:=\operatorname{ord}_{R_{1}}$, where

$$
w\left(x_{1}\right)=1 \quad \text { and } \quad w\left(x_{i}-a_{i} x_{1}\right)=2 \quad \text { for } \quad i=2, \ldots, d,
$$

and the images of $\frac{x_{2}-a_{2} x_{1}}{x_{1}^{2}}, \ldots, \frac{x_{d}-a_{d} x_{1}}{x_{1}^{2}}$ in $k_{w}$ are algebraically independent over $R_{0} / \mathbf{m}_{0}$. Thus Rees $P_{R_{0} R_{1}}=\operatorname{Rees}_{R_{1}} \mathbf{m}_{1}$.

(2) $\mathcal{B P}\left(P_{R_{0} R_{1}}\right)=\left\{R_{0}, R_{1}\right\}$.

(3) $\mathcal{B}\left(P_{R_{0} R_{1}}\right)=\{1,1\}$.

(4) The ideal $I:=P_{R_{0} R_{1}}$ is a normal ideal cf. [Go]. Hence the Rees algebra $R[I t]$ is a normal domain. Also $\frac{R[I t]}{Q} \cong\left(\frac{R}{\mathbf{m}}\right)\left[T_{1}, \ldots, T_{d}\right]$ is a polynomial ring in $d$-variables over $R / \mathbf{m}$, where $\operatorname{Min}(\mathbf{m} R[I t])=\{Q\}$ and $Q=\mathbf{m} R[I t]$. 
As a consequence of Proposition 6.4 and Remark 6.5, we have

Corollary 6.6. Let the notation be as in Proposition 6.4. Assume that $R_{0} / \mathbf{m}_{0}=$ $R_{n} / \mathbf{m}_{n}$. Then we have

$$
\left\{V_{n}\right\} \subseteq \text { Rees } P_{R_{0} R_{n}} \subseteq\left\{V_{0}, V_{1}, \ldots, V_{n-2}, V_{n}\right\} .
$$

With notation as in Setting 6.1, we illustrate in Example 6.7 the structure of the special $*$-simple complete ideal $I=P_{R_{0} R_{n}}$ in the case where $R_{0} / \mathbf{m}_{0}=R_{n} / \mathbf{m}_{n}$ and there is no change of direction. We assume $\operatorname{dim} R_{0}=3$. The situation is similar for $\operatorname{dim} R_{0}>3$.

Example 6.7. Let $\left(R, \mathbf{m}_{0}, k\right)$ be a 3 -dimensional regular local ring with maximal ideal $\mathbf{m}_{0}=(x, y, z) R$, and let $a_{1}, \ldots, a_{n}$ and $b_{1}, \ldots, b_{n}$ be elements in $R$. Consider the following finite sequence of local quadratic transformations

$$
R=: R_{0} \subset{ }^{x} R_{1} \subset{ }^{x x} R_{2} \subset{ }^{x x x} R_{3} \subset \cdots \subset^{\overbrace{}^{n \text { times }}} R_{n},
$$

where for $i=0,1,2, \ldots, n-1$ we define $S_{i+1}$ and $\overbrace{x \cdots x}^{i+1 \text { times }} R_{i+1}=R_{i+1}$ inductively by

$S_{1}:=R_{0}\left[\frac{\mathbf{m}_{0}}{x}\right], \quad N_{1}:=\left(x, \frac{y-a_{1} x}{x}, \frac{z-b_{1} x}{x}\right) S_{1} \quad R_{1}:=\left(S_{1}\right)_{N_{1}} \quad \mathbf{m}_{1}:=N_{1} R_{1}$

$S_{i+1}:=R_{i}\left[\frac{\mathbf{m}_{i}}{x}\right], \quad N_{i+1}:=\left(x, \frac{y-a_{1} x-\cdots-a_{i+1} x^{i+1}}{x^{i+1}}, \frac{z-b_{1} x-\cdots-b_{i+1} x^{i+1}}{x^{i+1}}\right) S_{i+1}$ $R_{i+1}:=\left(S_{i+1}\right)_{N_{i+1}} \quad \mathbf{m}_{i+1}:=N_{i+1} R_{i+1}$.

Then for $i=0,1, \ldots, n$, we have

(1) The order valuation $v_{i}:=\operatorname{ord}_{R_{i}}$ has values $v_{i}(x)=1$ and

$$
v_{i}\left(\frac{y-a_{1} x-\cdots-a_{i} x^{i}}{x^{i}}\right)=v_{i}\left(\frac{z-b_{1} x-\cdots-b_{i} x^{i}}{x^{i}}\right)=1 .
$$

and the images of

$$
\frac{y-a_{1} x-\cdots-a_{i} x^{i}}{x^{i+1}} \text { and } \frac{z-b_{1} x-\cdots-b_{i} x^{i}}{x^{i+1}}
$$

in the residue field $k_{v_{i}}$ of $V_{i}$ are algebraically independent over $R_{0} / \mathbf{m}_{0}$.

(2) The special $*$-simple complete $\mathbf{m}$-primary ideal is

$$
P_{R_{0} R_{i}}=\left(x^{i+1}, y-a_{1} x-\cdots-a_{i} x^{i}, z-b_{1} x-\cdots-b_{i} x^{i}\right) R .
$$

(3) $\mathcal{B P}\left(P_{R_{0} R_{i}}\right)=\left\{R_{0}, R_{1}, R_{2}, \ldots, R_{i}\right\}$.

(4) $\mathcal{B}\left(P_{R_{0} R_{i}}\right)=\{1,1,1, \ldots, 1\}$. 
(5) The special $*$-simple complete $\mathbf{m}$-primary ideal $P_{R_{0} R_{i}}$ has a unique Rees valuation $\operatorname{ord}_{R_{i}}$. That is, $\operatorname{Rees}\left(P_{R_{0} R_{i}}\right)=\operatorname{Rees}_{R_{i}} \mathbf{m}_{i}$.

(6) The ideal $P_{R_{0} R_{i}}$ is normal.

(7) Let $I:=P_{R_{0} R_{i}}$. Then $\mathbf{m} R[I t]$ has a unique minimal prime $Q:=\mathbf{m} R[I t]$ and $\frac{R[I t]}{Q}$ is a polynomial ring in 3 -variables over $R / \mathbf{m}$.

Theorem 6.8. Let the notation be as in Setting 6.1. Assume that $R_{0} / \mathbf{m}_{0}=$ $R_{n} / \mathbf{m}_{n}$. Then the following are equivalent:

(1) Rees $P_{R_{0} R_{n}}=\operatorname{Rees}_{R_{n}} \mathbf{m}_{n}$, i.e., $\operatorname{ord}_{R_{n}}$ is the unique Rees valuation of $P_{R_{0} R_{n}}$.

(2) Either $\operatorname{dim} R_{0}=2$, or there is no change of direction in the local quadratic sequence given in Equation 6 .

Proof. (2) $\Longrightarrow(1)$ : If $\operatorname{dim} R_{0}=2$, then the theory of Zariski implies that $I$ has a unique Rees valuation. Assume that $\operatorname{dim} R_{0} \geq 3$ and that there is no change of direction in the sequence given in Equation 6. By Example 6.7, we have that the special $*$-simple complete ideal $P_{R_{0} R_{n}}$ has the unique Rees valuation, $\operatorname{ord}_{R_{n}}$.

$(1) \Longrightarrow(2)$ : First, notice that $\operatorname{Rees}_{R_{n}} \mathbf{m}_{n} \subseteq$ Rees $P_{R_{0} R_{n}}$ by Proposition 6.4, and hence $\mid$ Rees $P_{R_{0} R_{n}} \mid \geq 1$. To conclude the proof, we prove the following :

Claim 6.9. If there is at least one change of direction in the local quadratic sequence given in Equation 6, then $\mid$ Rees $P_{R_{0} R_{n}} \mid>1$.

Proof. Assume there is at least one change of direction between $R_{0}$ and $R_{n}$. Choose $j$ minimal so that there is no change of direction from $R_{j+1}$ to $R_{n}$. Then by choosing appropriate regular parameters $x$ in $R_{j}$ and $y$ in $R_{j+1}$, we have the following local quadratic sequence:

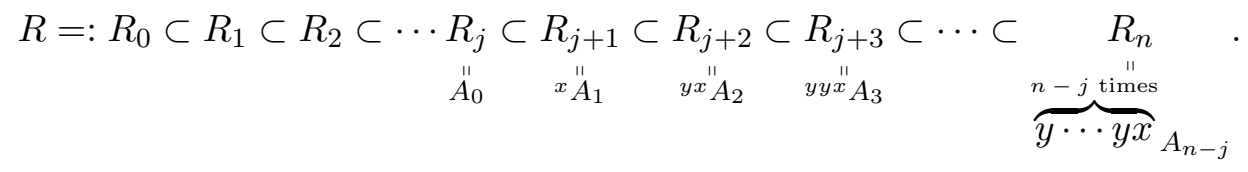

By Remark 6.3, we have $\operatorname{Rees}_{R_{j}} P_{R_{j} R_{n}} \subseteq$ Rees $P_{R_{0} R_{n}}$. Thus to complete the proof of the Claim, we analyse in Example 6.10 the structure of a special $*$-simple complete m-primary ideal of a $d \geq 3$-dimensional regular local ring obtained by a change of direction first dividing by $x$ and then successively by $y$. For notational simplicity, we assume that $d=3$. The pattern is similar in the case where $d>3$. 
Example 6.10. Let $(R, \mathbf{m}, k)$ be a 3 -dimensional regular local ring with maximal ideal $\mathbf{m}=(x, y, z) R$. Let $n \geq 3$. Consider a sequence of local quadratic transforms $R:=R_{0} \subset R_{1}:={ }^{x} R_{1} \subset R_{2}:={ }^{y x} R_{2} \subset R_{3}:={ }^{y y x} R_{3} \subset \cdots \subset R_{n}:=\overbrace{y \cdots y y x}^{n \text { times }} R_{n}$ defined by

$$
\begin{array}{lll}
S_{1}:=R\left[\frac{\mathbf{m}}{x}\right], & N_{1}:=\left(x, \frac{y}{x}, \frac{z}{x}\right) S_{1}, & R_{1}:=\left(S_{1}\right)_{N_{1}}, \mathbf{m}_{1}:=N_{1} R_{1} \\
S_{2}:=R_{1}\left[\frac{\mathbf{m}_{1}}{y / x}\right], & N_{2}:=\left(\frac{x^{2}}{y}, \frac{y}{x}, \frac{z-b_{2} y}{y}\right) S_{2}, & R_{2}:=\left(S_{2}\right)_{N_{2}}, \mathbf{m}_{2}:=N_{2} R_{2} \\
S_{3}:=R_{2}\left[\frac{\mathbf{m}_{2}}{y / x}\right], & N_{3}:=\left(\frac{x^{3}-a_{3} y^{2}}{y^{2}}, \frac{y}{x}, \frac{x z-b_{2} x y-b_{3} y^{2}}{y^{2}}\right) S_{3}, & R_{3}:=\left(S_{3}\right)_{N_{3}}, \mathbf{m}_{3}:=N_{3} R_{3} \\
\ldots & & R_{n}:=\left(S_{n}\right)_{N_{n}}, \mathbf{m}_{n}:=N_{n} R_{n}, \\
S_{n}:=R_{n-1}\left[\frac{\mathbf{m}_{n-1}}{y / x}\right], & N_{n}:=(f, g, h) S_{n}, &
\end{array}
$$

where

$$
\begin{aligned}
f & :=\frac{x^{n}-a_{3} x^{n-3} y^{2}-\cdots-a_{n-1} x y^{n-2}-a_{n} y^{n-1}}{y^{n-1}} \\
g & :=\frac{y}{x} \\
h & :=\frac{x^{n-2} z-b_{2} x^{n-2} y-\cdots-b_{n-1} x y^{n-2}-b_{n} y^{n-1}}{y^{n-1}} .
\end{aligned}
$$

Here the elements $a_{i}$ and $b_{j}$ are in $R_{0}$, and we are assuming that $R_{0} / \mathbf{m}_{0}=R_{n} / \mathbf{m}_{n}$. Thus we may choose $x, y, z$ so that $N_{1}=\left(x, \frac{y}{x}, \frac{z}{x}\right) S_{1}$. We are also assuming that there is a change of direction from $R_{0}$ to $R_{2}$. Thus we may assume $N_{2}=\left(\frac{x^{2}}{y}, \frac{y}{x}, \frac{z-b_{2} y}{y}\right) S_{2}$.

Let

$$
\begin{aligned}
& f_{0}:=x^{n}-a_{3} x^{n-3} y^{2}-\cdots-a_{n-1} x y^{n-2}-a_{n} y^{n-1} \\
& h_{0}:=x^{n-2} z-b_{2} x^{n-2} y-\cdots-b_{n-1} x y^{n-2}-b_{n} y^{n-1} .
\end{aligned}
$$

Then:

(1) Let $v_{n}:=\operatorname{ord}_{R_{n}}$. We have

$$
\begin{aligned}
v_{n}\left(f_{0}\right) & =1+(n-1) v_{n}(y), \\
v_{n}(y) & =1+v_{n}(x), \\
v_{n}\left(h_{0}\right) & =1+(n-1) v_{n}(y) .
\end{aligned}
$$

(2) Let

$$
K:=\left\{\alpha \in \mathbf{m}_{0} \mid v_{n}(\alpha) \geq v_{n}\left(y^{n}\right) \text { and } v_{0}(\alpha) \geq n\right\} .
$$

Then we have $K=P_{R_{0}} R_{n}$

(3) $\mathcal{B P}\left(P_{R_{0} R_{n}}\right)=\left\{R_{0}, R_{1}, R_{2}, R_{3} \ldots, R_{n}\right\}$.

(4) $\mathcal{B}\left(P_{R_{0} R_{n}}\right)=\{n, 1,1, \ldots, 1,1\}$. 
(5) The Rees valuations of $P_{R_{0} R_{n}}$ are $\operatorname{ord}_{R_{0}}$ and $\operatorname{ord}_{R_{n}}$.

Proof. For item (1), since $\mathbf{m}_{n}=(f, g, h) R_{n}$ we have

$$
\begin{aligned}
1 & =v_{n}\left(\frac{x^{n}-a_{3} x^{n-3} y^{2}-\cdots-a_{n-1} x y^{n-2}-a_{n} y^{n-1}}{y^{n-1}}\right)=v_{n}\left(\frac{y}{x}\right) \\
& =v_{n}\left(\frac{x^{n-2} z-b_{2} x^{n-2} y-\cdots-b_{n-1} x y^{n-2}-b_{n} y^{n-1}}{y^{n-1}}\right),
\end{aligned}
$$

and hence

$$
v_{n}\left(f_{0}\right)=1+(n-1) v_{n}(y), \quad v_{n}(y)=1+v_{n}(x), \quad v_{n}\left(h_{0}\right)=1+(n-1) v_{n}(y) .
$$

Multiplying the listed generators of $\mathbf{m}_{n}$ by $x y^{n-1}$, we obtain elememts in $R_{0}$

$$
x \cdot f_{0}, \quad y^{n}, \quad x \cdot h_{0}
$$

By Equation 7, we have

$$
n\left(1+v_{n}(x)\right)=v_{n}\left(x f_{0}\right)=v_{n}\left(y^{n}\right)=v_{n}\left(x h_{0}\right) .
$$

For item (2), we clearly have $K \supseteq\left(x f_{0}, y^{n}, x h_{0}\right) R_{0}$.

We observe that $v_{n}(z) \geq v_{n}(y)$; for if $v_{n}(z)<v_{n}(y)$, then

$$
v_{n}\left(x^{n-1} z\right)<v_{n}\left(-b_{2} x^{n-1} y-\cdots-b_{n} x y^{n-1}\right)
$$

implies that $v_{n}\left(x^{n-1} z\right)=v_{n}\left(x h_{0}\right)=v_{n}\left(y^{n}\right)$, a contradiction. Therefore $z^{n} \in K$.

Since $K$ has order $n$ and contains $y^{n}$ and $z^{n}$, we see that the transform of $K$ in $R_{0}\left[\frac{\mathbf{m}_{0}}{y}\right]$ and in $R_{0}\left[\frac{\mathbf{m}_{0}}{z}\right]$ is the whole ring. Let $y_{1}:=\frac{y}{x}$ and $z_{1}:=\frac{z}{x}$, then $y=x y_{1}$ and $z=x z_{1}$. Hence

$$
\begin{aligned}
& K R_{0}\left[\frac{\mathbf{m}_{0}}{x}\right]=K S_{1} \\
& \supseteq x^{n}\left(x-\left(a_{3} y_{1}^{2}-\cdots-a_{n} y_{1}^{n-1}\right), y_{1}^{n}, z_{1}-\left(b_{2} y_{1}-b_{3} y_{1}^{2}-\cdots-b_{n} y_{1}^{n-1}\right) S_{1} .\right.
\end{aligned}
$$

Thus the transform of $K$ in $S_{1}$ is

$$
K^{S_{1}} \supseteq\left(x-\left(a_{3} y_{1}^{2}-\cdots-a_{n} y_{1}^{n-1}\right), y_{1}^{n}, z_{1}-\left(b_{2} y_{1}-b_{3} y_{1}^{2}-\cdots-b_{n} y_{1}^{n-1}\right)\right) S_{1} .
$$

Since the ideal $K^{S_{1}}$ is primary for the maximal ideal $N_{1}=\left(x_{1}, y_{1}, z_{1}\right) S_{1}$ and $R_{1}=$ $\left(S_{1}\right)_{N_{1}}$, we have

$$
K^{S_{1}}=\left(x-\left(a_{3} y_{1}^{2}-\cdots-a_{n} y_{1}^{n-1}\right), y_{1}^{n}, z_{1}-\left(b_{2} y_{1}-b_{3} y_{1}^{2}-\cdots-b_{n} y_{1}^{n-1}\right)\right) S_{1},
$$

and hence

$$
K^{R_{1}}=\left(x-\left(a_{3} y_{1}^{2}-\cdots-a_{n} y_{1}^{n-1}\right), y_{1}^{n}, z_{1}-\left(b_{2} y_{1}-b_{3} y_{1}^{2}-\cdots-b_{n} y_{1}^{n-1}\right)\right) R_{1} .
$$

As in Example 6.7, we have

$$
P_{R_{1} R_{n}}=\left(x-\left(a_{3} y_{1}^{2}-\cdots-a_{n} y_{1}^{n-1}\right), y_{1}^{n}, z_{1}-\left(b_{2} y_{1}-b_{3} y_{1}^{2}-\cdots-b_{n} y_{1}^{n-1}\right)\right) R_{1},
$$


and thus $K^{R_{1}}=P_{R_{1} R_{n}}$. By $\left[\mathrm{L}\right.$, Proposition 2.1], we have $K=P_{R_{0} R_{n}}$. Items (3) and (4) are clear. Since $K$ has order $n$ and contains $y^{n}, z^{n}$ and $x h_{0}$, we see that $\operatorname{ord}_{R_{0}}$ is a Rees valuation of $K$. Therefore $\operatorname{ord}_{R_{0}}$ and $\operatorname{ord}_{R_{n}}$ are the Rees valuations of $K$.

This completes the proof of Theorem 6.8,

We illustrate in Examples 6.11 and 6.12 the behavior of a special $*$-simple complete ideal $P_{R_{0} R_{1}}$ in cases where $\left[R_{1} / \mathbf{m}_{1}: R_{0} / \mathbf{m}_{0}\right]>1$. In Example 6.11 the ideal $P_{R_{0} R_{1}}$ has two Rees valuations, while in Example 6.12 the ideal $P_{R_{0} R_{1}}$ has only one Rees valuation. We use notation as in Remark 6.5 with $d=3$ and $\mathbf{m}_{0}=(x, y, z) R_{0}$.

Example 6.11. Let $R_{0} / \mathbf{m}_{0}=\mathbb{Q}$ and $R_{1}:=\left(S_{1}\right)_{N_{1}}$, where

$$
N_{1}:=\left(x, \frac{y}{x},\left(\frac{z}{x}\right)^{2}-3\right) S_{1} \text {. }
$$

Let $w:=\operatorname{ord}_{R_{1}}$. Then we have

(1)

$$
w(x)=w\left(\frac{y}{x}\right)=w\left(\left(\frac{z}{x}\right)^{2}-3\right)=1,
$$

and the images of $\frac{y}{x^{2}}, \frac{z^{2}-3 x^{2}}{x^{3}}$ in the residue field $k_{w}$ of $w$ are algebraically independent over $R_{0} / \mathbf{m}_{0}$. Also $w\left(z^{2}-3 x^{2}\right)=1+w\left(x^{2}\right)=3$. Therefore

$$
\begin{array}{c|c|c|c|c} 
& x & y & z & z^{2}-3 x^{2} \\
\hline w:=\operatorname{ord}_{R_{1}} & 1 & 2 & 1 & 3
\end{array}
$$

(2) Let

$$
I:=\left\{\alpha \in \mathbf{m}_{0} \mid w(\alpha) \geq 3\right\}
$$

Then we have

(a) $I=\left(x^{3}, x y, z^{2}-3 x^{2}, y^{2}, y z, z^{3}\right) R_{0}$. A direct computation shows that $I=P_{R_{0} R_{1}}$. We have

\begin{tabular}{c|c|c|c|c|c|c}
$P_{R_{0} R_{1}}$ & $x^{3}$ & $x y$ & $z^{2}-3 x^{2}$ & $y^{2}$ & $y z$ & $z^{3}$ \\
\hline$w:=\operatorname{ord}_{R_{1}}$ & 3 & 3 & 3 & 4 & 3 & 3 \\
\hline$v:=\operatorname{ord}_{R_{0}}$ & 3 & 2 & 2 & 2 & 2 & 3
\end{tabular}

(b) $\mathcal{B P}\left(P_{R_{0} R_{1}}\right)=\left\{R_{0}, R_{1}\right\}$.

(c) $\mathcal{B}\left(P_{R_{0} R_{1}}\right)=\{2,1\}$.

(d) The set of Rees valuations of $P_{R_{0} R_{1}}$ is $\left\{\operatorname{ord}_{R_{0}}, \operatorname{ord}_{R_{1}}\right\}$.

Example 6.12. Let $R_{0} / \mathbf{m}_{0}=\mathbb{Q}$ and $R_{1}:=\left(S_{1}\right)_{N_{1}}$, where

$$
N_{1}:=\left(x,\left(\frac{y}{x}\right)^{2}-2,\left(\frac{z}{x}\right)^{2}-3\right) S_{1} .
$$

Let $w:=\operatorname{ord}_{R_{1}}$. Then we have 
(1)

$$
w(x)=w\left(\left(\frac{y}{x}\right)^{2}-2\right)=w\left(\left(\frac{z}{x}\right)^{2}-3\right)=1,
$$

and the images of $\frac{y^{2}-2 x^{2}}{x^{3}}, \frac{z^{2}-3 x^{2}}{x^{3}}$ in the residue field $k_{w}$ of $w$ are algebraically independent over $R_{0} / \mathbf{m}_{0}$. Also $w\left(y^{2}-2 x^{2}\right)=w\left(z^{2}-3 x^{2}\right)=1+w\left(x^{2}\right)=3$.

Therefore

$$
\begin{array}{l|l|l|l} 
& x & y & z \\
\hline w:=\operatorname{ord}_{R_{1}} & 1 & 1 & 1
\end{array}
$$

(2) Let

$$
I:=\left\{\alpha \in \mathbf{m}_{0} \mid w(\alpha) \geq 3\right\}
$$

Then we have

(a) $I=\left(y^{2}-2 x^{2}, z^{2}-3 x^{2}, \mathbf{m}_{0}^{3}\right) R_{0}$. A direct computation shows that $I=P_{R_{0} R_{1}}$. We have

\begin{tabular}{c|c|c|c|}
$P_{R_{0} R_{1}}$ & $y^{2}-2 x^{2}$ & $z^{2}-3 x^{2}$ & $\mathbf{m}_{0}^{3}$ \\
\hline$w:=\operatorname{ord}_{R_{1}}$ & 3 & 3 & 3 \\
\hline$v:=\operatorname{ord}_{R_{0}}$ & 2 & 2 & 3
\end{tabular}

(b) $\mathcal{B P}\left(P_{R_{0} R_{1}}\right)=\left\{R_{0}, R_{1}\right\}$.

(c) $\mathcal{B}\left(P_{R_{0} R_{1}}\right)=\{2,1\}$.

(d) $\operatorname{Rees}\left(P_{R_{0} R_{1}}\right)=\operatorname{Rees}_{R_{1}} \mathbf{m}_{1}$.

Example 6.13 illustrates a pattern with exactly one change of direction from $R_{0}$ to $R_{2}$ where $R_{0} / \mathbf{m}_{0}=R_{2} / \mathbf{m}_{2}$.

Example 6.13. Let $(R, \mathbf{m}, k)$ be a 3 -dimensional regular local ring with maximal ideal $\mathbf{m}=(x, y, z) R$. Consider the following sequence of local quadratic transforms

$$
R:=R_{0} \subset R_{1}:={ }^{x} R_{1} \subset R_{2}:={ }^{y x} R_{2}
$$

defined by

$$
\begin{aligned}
S_{1}:=R\left[\frac{\mathbf{m}}{x}\right], & N_{1}:=\left(x, \frac{y}{x}, \frac{z}{x}\right) S_{1}, & R_{1}:=\left(S_{1}\right)_{N_{1}}, \quad \mathbf{m}_{1}:=N_{1} R_{1} \\
S_{2}:=R_{1}\left[\frac{\mathbf{m}_{1}}{y / x}\right], & N_{2}:=\left(\frac{x^{2}}{y}, \frac{y}{x}, \frac{z-b_{2} y}{y}\right) S_{2}, & R_{2}:=\left(S_{2}\right)_{N_{2}}, \quad \mathbf{m}_{2}:=N_{2} R_{2}
\end{aligned}
$$

Then:

(1) Let $v_{2}:=\operatorname{ord}_{R_{2}}$. Then $v_{2}(x)=2, v_{2}(y)=3, v_{2}\left(z-b_{2} y\right)=4$, and the images of $\frac{x^{3}}{y^{2}}, \frac{x\left(z-b_{2} y\right)}{y^{2}}$ in the residue field $k_{v_{2}}$ of $V_{2}$ are algebraically independent over $R_{2} / \mathbf{m}_{2}=k$. 
(2) The special $*$-simple complete $\mathbf{m}$-primary ideal $P_{R_{0} R_{2}}$ is a $v_{2}$-ideal. We have

$$
\begin{aligned}
P_{R_{0} R_{2}} & =\left\{\alpha \in \mathbf{m} \mid v_{2}(\alpha) \geq 6\right\} \\
& =\left(x^{3}, x\left(z-b_{2} y\right), y^{2}, x^{2} y, y\left(z-b_{2} y\right),\left(z-b_{2} y\right)^{2}\right) R .
\end{aligned}
$$

(3) $\mathcal{B P}\left(P_{R_{0} R_{2}}\right)=\left\{R_{0}, R_{1}, R_{2}\right\}$.

(4) $\mathcal{B}\left(P_{R_{0} R_{2}}\right)=\{2,1,1\}$.

(5) The set of Rees valuations of $P_{R_{0} R_{2}}$ is $\left\{\operatorname{ord}_{R_{0}}, \operatorname{ord}_{R_{2}}\right\}$.

(6) Let $I:=P_{R_{0} R_{2}}$. Then :

(a) $\operatorname{Min}(\mathbf{m} R[I t])=\left\{P_{0}, P_{2}\right\}$, where

$$
\begin{aligned}
P_{2} & =\left(\mathbf{m}, x^{2} y t, y\left(z-b_{2} y\right) t,\left(z-b_{2} y\right)^{2} t\right) R[I t] \quad \text { and } \\
P_{0} & =\left(\mathbf{m}, x^{3} t, x^{2} y t\right) R[I t] .
\end{aligned}
$$

(b) Let $V_{0}$ and $V_{2}$ denote the valuation rings corresponding to $v_{0}:=\operatorname{ord}_{R_{0}}$ and $v_{2}:=\operatorname{ord}_{R_{2}}$, where $V_{0}=R[I t]_{P_{0}} \cap \mathcal{Q}(R)$ and $V_{2}=R[I t]_{P_{2}} \cap \mathcal{Q}(R)$. Then $\frac{R[I t]}{P_{2}}$ is a polynomial ring in 3 -variables over $R / \mathbf{m}$, and $\frac{R[I t]}{P_{0}} \cong$ $\left.\frac{(R / \mathbf{m})\left[T_{1}, T_{2}, T_{3}, T_{4}\right]}{\left(T_{2} T_{4}-T_{3}^{2}\right)}\right)$ is a 3 -dimensional normal Cohen-Macaulay domain with minimal multiplicity at its maximal homogeneous ideal with this multiplicity being 2 .

Proof. (1) : Since $\mathbf{m}_{2}=\left(\frac{x^{2}}{y}, \frac{y}{x}, \frac{z-b_{2} y}{y}\right) R_{2}$, we have $v_{2}\left(\frac{x^{2}}{y}\right)=v_{2}\left(\frac{y}{x}\right)=v_{2}\left(\frac{z-b_{2} y}{y}\right)=1$, and hence $v_{2}(x)=2, v_{2}(y)=3, v_{2}\left(z-b_{2} y\right)=4$, and and the images of $\frac{x^{3}}{y^{2}}, \frac{x\left(z-b_{2} y\right)}{y^{2}}$ in the residue field $k_{v_{2}}$ of $V_{2}$ are algebraically independent over $R_{2} / \mathbf{m}_{2}=k$. (2), (3), and (4) : The transform in $R_{2}$ of the ideal $K:=\left(x^{3}, y^{2}, x\left(z-b_{2} y\right)\right) R$ is the maximal ideal $\mathbf{m}_{2}=\left(\frac{x^{2}}{y}, \frac{y}{x}, \frac{z-b_{2} y}{y}\right) R_{2}$ and $v_{2}(K)=6$. Let

$$
I:=\left\{\alpha \in \mathbf{m} \mid v_{2}(\alpha) \geq 6\right\}=\left(x^{3}, x\left(z-b_{2} y\right), y^{2}, x^{2} y, y\left(z-b_{2} y\right),\left(z-b_{2} y\right)^{2}\right) R .
$$

The ideal $I$ is a complete $\mathbf{m}$-primary ideal. We see by direct computation that:

(a) $I^{R_{1}}=\left(x,\left(\frac{y}{x}\right)^{2}, \frac{z-b_{2} y}{x}\right) R_{1}$ is a special $*$-simple complete ideal in $R_{1}$.

(b) $I^{R_{2}}=\mathbf{m}_{2}$.

(c) $\mathcal{B P}(I)=\left\{R_{0}, R_{1}, R_{2}\right\}$.

Thus by [L, Proposition 2.1], we have $I=P_{R_{0} R_{2}}$. It is clear that $\operatorname{ord}_{R_{0}}(I)=$ $2, \operatorname{ord}_{R_{1}}\left(I^{R_{1}}\right)=1, \operatorname{ord}_{R_{2}}\left(I^{R_{2}}\right)=1$. Hence $\mathcal{B}(I)=\{2,1,1\}$.

(5) : By item (1), $v_{2}:=\operatorname{ord}_{R_{2}}$ is a Rees valuation of $P_{R_{0} R_{2}}$. We have the following table :

\begin{tabular}{c|c|c|c|c|c|c|}
$P_{R_{0} R_{2}}$ & $x^{3}$ & $x\left(z-b_{2} y\right)$ & $y^{2}$ & $x^{2} y$ & $y\left(z-b_{2} y\right)$ & $\left(z-b_{2} y\right)^{2}$ \\
\hline$v_{2}:=\operatorname{ord}_{R_{2}}$ & 6 & 6 & 6 & 7 & 7 & 8 \\
\hline$v_{1}:=\operatorname{ord}_{R_{1}}$ & 3 & 3 & 4 & 4 & 4 & 4 \\
\hline$v_{0}:=\operatorname{ord}_{R_{0}}$ & 3 & 2 & 2 & 3 & 2 & 2
\end{tabular}


Since the images of $\frac{y^{2}}{y z}, \frac{x\left(z-b_{2} y\right)}{y z}$ in the residue field $k_{v_{0}}$ of $V_{0}$ are algebrically independent over $k, v_{0}:=\operatorname{ord}_{R_{0}}$ is a Rees valuation of $P_{R_{0} R_{2}}$. Using Proposition 6.4, we conclude that $P_{R_{0} R_{2}}$ has the two Rees valuations, $v_{0}:=\operatorname{ord}_{R_{0}}$ and $v_{2}:=\operatorname{ord}_{R_{2}}$.

(6) : The statements of item (6) follow from the previous items and the connection between the Rees valuations of $I$ and minimal primes of $\mathbf{m} R[I t]$ in the Rees algebra $R[I t]$, cf. [HK].

Example6.14illustrates a pattern where there are exactly two changes of direction from $R_{0}$ to $R_{3}$ and where $R_{0} / \mathbf{m}_{0}=R_{3} / \mathbf{m}_{3}$.

Example 6.14. Let $(R, \mathbf{m}, k)$ be a 3 -dimensional regular local ring with maximal ideal $\mathbf{m}=(x, y, z) R$. Consider the following sequence of local quadratic transforms

$$
R:=R_{0} \subset R_{1}:={ }^{x} R_{1} \subset R_{2}:={ }^{y x} R_{2} \subset R_{3}:={ }^{z y x} R_{3}
$$

defined by

$$
\begin{aligned}
S_{1}:=R\left[\frac{\mathbf{m}}{x}\right], & N_{1}:=\left(x, \frac{y}{x}, \frac{z}{x}\right) S_{1}, & R_{1}:=\left(S_{1}\right)_{N_{1}}, & \mathbf{m}_{1}:=N_{1} R_{1} \\
S_{2}:=R_{1}\left[\frac{\mathbf{m}_{1}}{y / x}\right], & N_{2}:=\left(\frac{x^{2}}{y}, \frac{y}{x}, \frac{z}{y}\right) S_{2}, & R_{2}:=\left(S_{2}\right)_{N_{2}}, & \mathbf{m}_{2}:=N_{2} R_{2} \\
S_{3}:=R_{2}\left[\frac{\mathbf{m}_{2}}{z / y}\right], & N_{3}:=\left(\frac{x^{2}}{z}, \frac{y^{2}}{x z}, \frac{z}{y}\right) S_{2}, & R_{3}:=\left(S_{3}\right)_{N_{3}}, & \mathbf{m}_{3}:=N_{3} R_{3} .
\end{aligned}
$$

Then:

(1) Let $w_{3}:=\operatorname{ord}_{R_{3}}$. Then $w_{3}(x)=4, w_{3}(y)=6, w_{3}(z)=7$, and the images of $\frac{x^{2} y}{z^{2}}, \frac{y^{3}}{x z^{2}}$ in the residue field $k_{w_{3}}$ of $W_{3}$ are algebraically independent over $R_{3} / \mathbf{m}_{3}=k$.

(2) The special $*$-simple complete $\mathbf{m}$-primary ideal $P_{R_{0} R_{3}}$ is a $w_{3}$-ideal. We have

$$
\begin{aligned}
P_{R_{0} R_{3}} & =\left\{\alpha \in \mathbf{m} \mid w_{3}(\alpha) \geq 18\right\} \\
& =\left(x^{3} y, y^{3}, x z^{2}, y^{2} z, x^{3} z, x^{5}, y z^{2}, x^{2} y^{2}, z^{3}, x^{2} y z\right) R .
\end{aligned}
$$

(3) $\mathcal{B P}\left(P_{R_{0} R_{3}}\right)=\left\{R_{0}, R_{1}, R_{2}, R_{3}\right\}$.

(4) $\mathcal{B}\left(P_{R_{0} R_{3}}\right)=\{3,2,1,1\}$.

(5) The set of Rees valuations of $P_{R_{0} R_{3}}$ is $\left\{\operatorname{ord}_{R_{0}}, \operatorname{ord}_{R_{1}}, \operatorname{ord}_{R_{3}}\right\}$.

(6) Let $I:=P_{R_{0} R_{3}}$. Then :

(a) $\operatorname{Min}(\mathbf{m} R[I t])=\left\{Q_{0}, Q_{1}, Q_{3}\right\}$, where

$$
\begin{aligned}
Q_{3} & =\left(\mathbf{m}, y^{2} z t, x^{3} z t, x^{5} t, y z^{2} t, x^{2} y^{2} t, z^{3} t, x^{2} y z t\right) R[I t] \\
Q_{1} & =\left(\mathbf{m}, y^{3} t, y^{2} z t, y z^{2} t, x^{2} y^{2} t, z^{3} t, x^{2} y z t\right) R[I t] \\
Q_{0} & =\left(\mathbf{m}, x^{3} y t, x^{3} z t, x^{5} t, x^{2} y^{2} t, x^{2} y z t\right) R[I t] .
\end{aligned}
$$


(b) Let $W_{i}$ denote the valuation rings corresponding to $w_{i}:=\operatorname{ord}_{R_{i}}$, where $W_{i}=R[I t]_{Q_{i}} \cap \mathcal{Q}(R)$ for $i=0,1,3$. Then $\frac{R[I t]}{Q_{3}}$ is a polynomial ring in 3-variables over $R / \mathbf{m}$, and $\frac{R[I t]}{Q_{1}} \cong \frac{(R / \mathbf{m})\left[T_{1}, T_{2}, T_{3}, T_{4}\right]}{\left(T_{2} T_{4}-T_{3}^{2}\right)}$ is a 3 dimensional normal Cohen-Macaulay domain with minimal multiplicity at its maximal homogeneous ideal with this multiplicity being 2 . $\frac{R[I t]}{Q_{0}} \cong \frac{(R / \mathbf{m})\left[T_{1}, T_{2}, T_{3}, T_{4}, T_{5}\right]}{\mathcal{J}}$ is a 3 -dimensional normal Cohen-Macaulay domain with minimal multiplicity at its maximal homogeneous ideal with this multiplicity being 3 , where the ideal $\mathcal{J}$ is generated by the $2 \times 2$ minors of the matrix $\left[\begin{array}{lll}T_{1} & T_{3} & T_{4} \\ T_{3} & T_{4} & T_{5}\end{array}\right]$.

Proof. (1) : Since $\mathbf{m}_{3}=\left(\frac{x^{2}}{z}, \frac{y^{2}}{x z}, \frac{z}{y}\right) R_{3}$, we have $w_{3}\left(\frac{x^{2}}{z}\right)=w_{3}\left(\frac{y^{2}}{x z}\right)=w_{3}\left(\frac{z}{y}\right)=1$, and hence $w_{3}(x)=4, w_{3}(y)=6, w_{3}(z)=7$, and the images of $\frac{x^{2} y}{z^{2}}, \frac{y^{3}}{x z^{2}}$ in the residue field $k_{w_{3}}$ of $W_{3}$ are algebraically independent over $R_{3} / \mathbf{m}_{3}=k$.

(2), (3), and (4): The transform in $R_{3}$ of the ideal $K:=\left(x^{3} y, y^{3}, x z^{2}\right) R$ is the maximal ideal $\mathbf{m}_{3}=\left(\frac{x^{2}}{z}, \frac{y^{2}}{x z}, \frac{z}{y}\right) R_{3}$ and $w_{3}(K)=18$. Let

$I:=\left\{\alpha \in \mathbf{m} \mid w_{3}(\alpha) \geq 18\right\}=\left(x^{3} y, y^{3}, x z^{2}, y^{2} z, x^{3} z, x^{5}, y z^{2}, x^{2} y^{2}, z^{3}, x^{2} y z\right) R$.

The ideal $I$ is a $w_{3}$-ideal. We see by direct computation that:

(a) $I^{R_{1}}=\left(\left(\frac{y}{x}\right)^{3}, y,\left(\frac{z}{x}\right)^{2},\left(\frac{y}{x}\right)^{2}\left(\frac{z}{x}\right), z, x^{2}\right) R_{1}=P_{R_{1} R_{3}}$.

(b) $I^{R_{2}}=K^{R_{2}}=\left(\frac{y}{x}, \frac{x^{2}}{y},\left(\frac{z}{y}\right)^{2}\right) R_{2}=P_{R_{2} R_{3}}$

(c) $I^{R_{3}}=\mathbf{m}_{3}$.

(d) $\mathcal{B P}(I)=\left\{R_{0}, R_{1}, R_{2}, R_{3}\right\}$.

Thus by $\left[\mathrm{L}\right.$, Proposition 2.1], we have $I=P_{R_{0} R_{3}}$. It is clear that $\operatorname{ord}_{R_{0}}(I)=$ $3, \operatorname{ord}_{R_{1}}\left(I^{R_{1}}\right)=2, \operatorname{ord}_{R_{2}}\left(I^{R_{2}}\right)=1, \operatorname{ord}_{R_{3}}\left(I^{R_{3}}\right)=1$. Hence $\mathcal{B}(I)=\{3,2,1,1\}$.

(5) : By item (1), $w_{3}:=\operatorname{ord}_{R_{3}}$ is a Rees valuation of $P_{R_{0} R_{3}}$. We have the following table :

\begin{tabular}{c|c|c|c|c|c|c|c|c|c|c|}
$P_{R_{0} R_{3}}$ & $x^{3} y$ & $y^{3}$ & $x z^{2}$ & $y^{2} z$ & $x^{3} z$ & $x^{5}$ & $y z^{2}$ & $x^{2} y^{2}$ & $z^{3}$ & $x^{2} y z$ \\
\hline$w_{3}:=\operatorname{ord}_{R_{3}}$ & 18 & 18 & 18 & 19 & 19 & 20 & 20 & 20 & 21 & 21 \\
\hline$w_{2}:=\operatorname{ord}_{R_{2}}$ & 9 & 9 & 10 & 10 & 10 & 10 & 11 & 10 & 12 & 11 \\
\hline$w_{1}:=\operatorname{ord}_{R_{1}}$ & 5 & 6 & 5 & 6 & 5 & 5 & 6 & 6 & 6 & 6 \\
\hline$w_{0}:=\operatorname{ord}_{R_{0}}$ & 4 & 3 & 3 & 3 & 4 & 5 & 3 & 4 & 3 & 4
\end{tabular}

Since the images of $\frac{x^{3} z}{x^{3} y}, \frac{x^{3} z}{x^{5}}$ in the residue field $k_{w_{1}}$ of $W_{1}$ are algebrically independent over $k, w_{1}:=\operatorname{ord}_{R_{1}}$ is a Rees valuation of $P_{R_{0} R_{3}}$, and also since the images of $\frac{x z^{2}}{z^{3}}, \frac{y^{2} z}{y^{3}}$ in the residue field $k_{w_{0}}$ of $W_{0}$ are algebrically independent over $k$, $w_{0}:=\operatorname{ord}_{R_{0}}$ is a Rees valuation of $P_{R_{0} R_{3}}$. Using Proposition 6.4, we conclude that $P_{R_{0} R_{3}}$ has the three Rees valuations, $\operatorname{ord}_{R_{0}}, \operatorname{ord}_{R_{1}}$, and $\operatorname{ord}_{R_{3}}$. 
(6) : The statements of item (6) follow from the previous items and the connection between the Rees valuations of $I$ and minimal primes of $\mathbf{m} R[I t]$ in the Rees algebra $R[I t]$, cf. [HK].

\section{REFERENCES}

[A] S.S. Abhyankar, On the valuations centered in a local domain, Amer. J. Math. 78 (1956) 321-348.

[CGL] A. Campillo, G. Gonzalez-Sprinberg, and M. Lejeune-Jalabert, Clusters of infinitely near points, Math. Ann., 306 (1996), 169-194.

[CHRR1] C. Ciuperca, W. Heinzer, L. Ratliff Jr., and D. Rush, Projective equivalent ideals and Rees valuations, J. Algebra, 282 (2004), 140-156.

[CHRR2] C. Ciuperca, W. Heinzer, L. Ratliff Jr., and D. Rush, Projective full ideals in Noetherian rings, a survey, Contemporary Math. 448 (2007), 33-42.

[C] S. Cutkosky, Factorization of complete ideals, J. Algebra, 115 (1988), 144-149.

[G1] J. Gately, Unique factorization of *-products of one-fibered monomial ideals, Comm. Algebra, 28 (2000), 3137-3153.

[G2] J. Gately, *-Simple complete monomial ideals, Comm. Algebra, 33 (2005), 2833-2849.

[Go] S. Goto, Integral closedness of complete-intersection ideals, J. Algebra, 108 (1987), 151160.

[HK] W. Heinzer and M.-K. Kim, Integrally closed ideals in regular local rings of dimension two, J. Pure Appl. Algebra 216 (2012), 1-11.

[Hu] C. Huneke, The primary components of and integral closures of ideals in 3-dimensional regular local rings, Math. Ann., 275 (1986), 617-635.

[HS] C. Huneke and J. Sally, Birational Extensions in Dimension Two and Integrally Closed Ideals, J. Algebra, 115 (1988), 481-500.

[K] M.-K. Kim, Product of distinct simple integrally closed ideals in 2-dimensional regular local rings, Proc. Amer. Math. Soc. 125 (1997), 315-321.

[L] J. Lipman, On complete ideals in regular local rings, Algebraic Geometry and Commutative Algebra in Honor of Masayoshi Nagata, (1986), 203-231.

[M] H. Matsumura, Commutative Ring Theory, Cambridge Univ. Press, Cambridge, 1986.

[MRS] S. McAdam, L.J. Ratliff, Jr., J.D. Sally, Integrally closed projectively equivalent ideals, in: Commutative Algebra, in: Math. Sci. Res.Inst.Publ. 15 (1988), 391-405.

[Nag] M. Nagata, Note on a paper of Samuel concerning asymptotic properties of ideals, Mem. Coll. Sci. Univ. Kyoto Ser. A Math. 30 (1957), 165-176.

[N] M. Nagata, Local Rings, Interscience, New York, 1962.

[Rees] D. Rees, Valuations associated with ideals (II), J. London Mat. Soc. 36 (1956), 221-228.

[Sa1] J. Sally, Regular overrings of regular local rings, Trans. Amer. Math. Soc. 171 (1972), 291-300. Erratum Trans. Amer. Math. Soc. 213 (1975), 429.

[Sa2] J. Sally, Fibers over closed points of birational morphisms of nonsingular varieties, Amer. J. Math. 104 (1980), 545-552.

[Sam] P. Samuel, Some asymptotic properties of powers of ideals, Ann. of Math. 56 (1952), 11-21.

[SH] I. Swanson and C. Huneke, Integral Closure of Ideals, Rings, and Modules, London Math. Soc. Lecture Note Series 336, Cambridge Univ. Press, Cambridge, 2006.

[ZS2] O. Zariski and P. Samuel, Commutative Algebra, Vol. 2, D. Van Nostrand, New York, 1960.

Department of Mathematics, Purdue University, West Lafayette, Indiana 47907 U.S.A.

E-mail address: heinzer@math.purdue.edu

Department of Mathematics, Sungkyunkwan University, Jangangu Suwon 440-746, KOREA

E-mail address: mkkim@skku.edu 